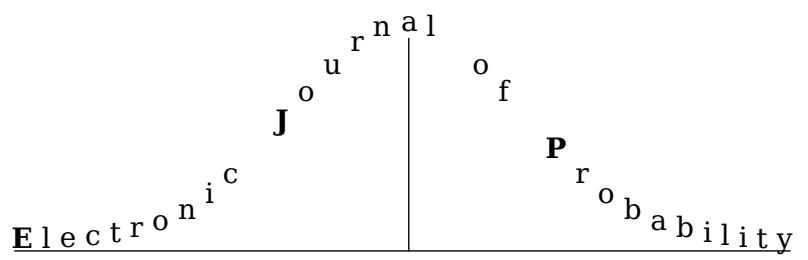

Electron. J. Probab. 26 (2021), article no. 64, 1-27.

ISSN: 1083-6489 https://doi.org/10.1214/21-EJP619

\title{
Backward stochastic differential equations with non-Markovian singular terminal conditions for general driver and filtration*
}

\author{
Mahdi Ahmadi ${ }^{\dagger} \quad$ Alexandre Popier ${ }^{\ddagger} \quad$ Ali Devin Sezer ${ }^{\S}$
}

\begin{abstract}
We consider a class of Backward Stochastic Differential Equations with superlinear driver process $f$ adapted to a filtration supporting at least a $d$ dimensional Brownian motion and a Poisson random measure on $\mathbb{R}^{m} \backslash\{0\}$. We consider the following class of terminal conditions: $\xi_{1}=\infty \cdot \mathbf{1}_{\left\{\tau_{1} \leq T\right\}}$ where $\tau_{1}$ is any stopping time with a bounded density in a neighborhood of $T$ and $\xi_{2}=\infty \cdot \mathbf{1}_{A_{T}}$ where $A_{t}, t \in[0, T]$ is a decreasing sequence of events adapted to the filtration $\mathcal{F}_{t}$ that is continuous in probability at $T$ (equivalently, $A_{T}=\left\{\tau_{2}>T\right\}$ where $\tau_{2}$ is any stopping time such that $\mathbb{P}\left(\tau_{2}=T\right)=0$ ). In this setting we prove that the minimal supersolutions of the BSDE are in fact solutions, i.e., they attain almost surely their terminal values. We note that the first exit time from a time varying domain of a $d$-dimensional diffusion process driven by the Brownian motion with strongly elliptic covariance matrix does have a continuous density. Therefore such exit times can be used as $\tau_{1}$ and $\tau_{2}$ to define the terminal conditions $\xi_{1}$ and $\xi_{2}$. The proof of existence of the density is based on the classical Green's functions for the associated PDE.
\end{abstract}

Keywords: backward stochastic differential equation; singularity; continuity problem; Green's function; density of hitting time.

MSC2020 subject classifications: 60G40; 60G99; 60H99; 65M80.

Submitted to EJP on April 30, 2020, final version accepted on March 29, 2021.

\section{Introduction and definitions}

A stochastic differential equation with a prescribed terminal condition is called a backward stochastic differential equation (BSDE). If a terminal condition can take the value $+\infty$ it is said to be singular. BSDE with singular terminal conditions has

\footnotetext{
*This work is supported by TUBITAK (The Scientific and Technological Research Council of Turkey) through project number 118F163. We are grateful for this support.

${ }^{\dagger}$ Institute of Applied Mathematics, Middle East Technical University. E-mail: mahdi.ahmadi@metu.edu.tr

${ }^{\ddagger}$ Laboratoire Manceau de Mathématiques, Le Mans Université, Avenue O. Messiaen, 72085 Le Mans cedex 9, France. E-mail: alexandre.popier@univ- lemans. $\mathrm{fr}$

${ }^{\S}$ Institute of Applied Mathematics, Middle East Technical University. E-mail: devin@metu.edu.tr
} 
received considerable attention at least since [33]. They generalize diffusion-reaction partial differential equations (PDE) where the singularity of the terminal condition of the BSDE corresponds to singularities in the final trace of the solution of the PDE (see $[17,33,34,35]$ and [26]). Moreover BSDE with a singularity at time $T$ is a useful tool in the solution of optimal stochastic control problems with terminal constraints (see $[1,17,23]$ and the references therein). This type of control problem can be interpreted as an optimal liquidation problem in finance (see the preceding references and [18] for an overview). Given a BSDE with a terminal condition $\xi$ at $T$, a process $Y$ satisfying the BSDE is said to be supersolution if

$$
\liminf _{t \rightarrow T} Y_{t} \geq \xi
$$

holds almost surely; $Y$ is said to be minimal if every other supersolution dominates it. As explained below, minimal supersolutions and their properties play a key role our analysis. We say $Y$ solves the BSDE with singular terminal condition $\xi$ if

$$
\lim _{t \rightarrow T} Y_{t}=\xi ;
$$

i.e., to go from a supersolution to a solution we need to replace the lim inf in (1.1) with lim and $\geq$ with $=$. In the rest of this paper whenever we refer to the "solution" of a BSDE with a singular terminal value, it will be in the sense of (1.2). The condition (1.2) means that the process $Y$ is continuous at time $T$; for this reason we refer to the problem of establishing that a candidate solution satisfies (1.2) as the "continuity problem." We further comment on the distinction between solutions (in the sense of (1.2)) and minimal supersolutions below. While minimal supersolutions of BSDE with singular terminal conditions is available in a general setting (see [23] and subsection 1.2 below), solutions of BSDE with singular terminal conditions are mostly available for Markovian terminal conditions, i.e., terminal conditions which are deterministic functions of an underlying adapted Markov process; see subsection 1.2 for a summary of known results.

The first work to solve a BSDE with a non-Markovian singular terminal condition was [39] treating the following problem:

$$
\begin{aligned}
Y_{t} & =Y_{s}-\int_{s}^{t} Y_{r}\left|Y_{r}\right|^{q-1} d r-\int_{s}^{t} Z_{r} d W_{r}, 0<s<t<T, \\
Y_{T} & =\xi,
\end{aligned}
$$

where $q>1$ and $W$ is a single dimensional Brownian motion,

$$
\xi=\infty \cdot \mathbf{1}_{\left\{\tau_{0} \leq T\right\}} \text { or } \xi=\infty \cdot \mathbf{1}_{\left\{\tau_{0}>T\right\}}
$$

and $\tau_{0}$ is the first exit time of $W$ from an interval $[a, b]$. The goal of the present work is to generalize these results in the following directions:

1. Work with a more general filtration supporting a $d$-dimensional Brownian motion and a Poisson random measure,

2. More general driver processes $f$ that is allowed to be an $\mathbb{F}$-adapted process,

3. For $\xi_{1}=\infty \cdot \mathbf{1}_{\{\tau \leq T\}}$ we allow $\tau$ to be any stopping time whose distribution around $T$ has a bounded density; we show that the exit time of a multidimensional continuous diffusion process from a time varying domain satisfies the density condition.

4. Extend $\xi_{2}=\infty \cdot \mathbf{1}_{\{\tau>T\}}$ to the more general terminal condition $\xi_{2}=\infty \cdot \mathbf{1}_{A_{T}}$ where $A_{t}, t \in[0, T]$ is a decreasing sequence of events adapted to $\mathcal{F}_{T}$ that is left continuous in probability at $T$. 
Let $\left(\Omega, \mathcal{F}, \mathbb{P}, \mathbb{F}=\left(\mathcal{F}_{t}\right)_{t \geq 0}\right)$ be a filtered probability space. The filtration $\mathbb{F}$ is assumed to be complete, right continuous, it supports a $d$ dimensional Brownian motion $W$ and a Poisson random measure $\pi$ with intensity $\mu(d e) d t$ on the space $\mathcal{E} \subset \mathbb{R}^{m} \backslash\{0\}$. The measure $\mu$ is $\sigma$-finite on $\mathcal{E}$ and satisfies

$$
\int_{\mathcal{E}}\left(1 \wedge|e|^{2}\right) \mu(d e)<+\infty .
$$

The compensated Poisson random measure $\widetilde{\pi}(d e, d t)=\pi(d e, d t)-\mu(d e) d t$ is a martingale with respect to the filtration $\mathbb{F}$. In this framework we will study the following generalization of (1.3):

$$
\begin{aligned}
Y_{t} & =Y_{s}+\int_{t}^{s} f\left(r, Y_{r}, Z_{r}, \psi_{r}\right) d r-\int_{t}^{s} Z_{r} d W_{r}-\int_{t}^{s} \int_{\mathcal{E}} \psi_{r}(e) \widetilde{\pi}(d e, d r)-\int_{t}^{s} d M_{r}, \\
Y_{T} & =\xi,
\end{aligned}
$$

$0 \leq t<s<T$. We call $(Y, Z, \psi, M)$ a solution to the $\operatorname{BSDE}(1.5,1.6)$ if $(Y, Z, \psi, M)$ satisfies $(1.5,1.6)$ and $Y$ is continuous at $T$, i.e.,

$$
\lim _{t \rightarrow T} Y_{t}=Y_{T}=\xi ;
$$

The driver $f$, generalizing the deterministic $-y|y|^{q-1}$ appearing in (1.3), is defined on $\Omega \times[0, T] \times \mathbb{R} \times \mathbb{R}^{k} \times \mathcal{B}_{\mu}^{2}\left(\mathcal{B}_{\mu}^{2}\right.$ is a functional space defined by (1.11)), and for any fixed $y$, $z, \psi, f(t, y, z, \psi)$ is assumed to be a progressively measurable process; thanks to a priori bounds and comparison results proved in $[22,23,24]$, we are able to work with a very general class of drivers; to be able to use their bounds and comparison results we will adopt the assumptions these works make on the filtration and on the driver, which are listed in subsection 1.2 as conditions (A) and (B).

In Section 2 we solve the BSDE $(1.5,1.6)$ with $^{1}$

$$
\xi=\xi_{1}=\infty \cdot \mathbf{1}_{\{\tau \leq T\}},
$$

where $\tau$ is any stopping time whose distribution in a neighborhood of $T$ has a bounded density. In Section 3 we treat terminal conditions of the form

$$
\xi=\xi_{2}=\infty \cdot \mathbf{1}_{A_{T}},
$$

where $A_{t}$ is a decreasing left continuous sequence of events adapted to our filtration that is left continuous in probability at time $T$ :

$$
\mathbb{P}\left(\bigcap_{t<T} A_{t} \backslash A_{T}\right)=0 .
$$

Lemma 3.2 of Section 3 shows that the formulation of the terminal condition $\xi_{2}$ in terms of a decreasing sequence of events is equivalent to setting $\{\tau>T\}$ where $\tau$ is a stopping time with $\mathbb{P}(\tau=T)=0$.

We know from [23] that the BSDE (1.5) has a minimal supersolution $Y_{t}^{\min }$ with terminal condition $\xi_{1}$. The goal of Section 2 is to prove that $Y_{t}^{\min }$ is continuous at $T$ and has $\xi_{1}$ as its limit- this implies that the supersolution is indeed a solution. Let $Y^{\infty}$ be the solution of (1.5) with terminal condition $\xi=\infty$ identically. The main idea in establishing the continuity of the minimal supersolution is to use the solution of a linear BSDE with terminal condition $Y_{\tau}^{\infty} \cdot \mathbf{1}_{\{\tau \leq T\}}$ as an upper bound on the time interval $[0, \tau \wedge T]$ (see

\footnotetext{
${ }^{1}$ We define $0 \cdot \infty:=0$.
} 
(2.2) and (2.3)). The proof that the upperbound process is well defined involves two ingredients 1) the fact that $\tau$ has a density and 2) a priori upperbounds on $Y^{\infty}$ derived in [23]. Although the approach of [39] is different from the one outlined above, it uses these ingredients as well, both of which are elementary in the setup treated in [39]: there is an explicit formula for the density of the exit time $\tau_{0}$ and the process $t \mapsto y_{t}$ in [39] corresponding to $Y^{\infty}$ is deterministic with an elementary formula so no a priori bounds were needed in [39].

The treatment of $\xi_{2}$ given in Section 3 is a generalization of the argument given in [39] dealing with $\infty \cdot 1_{\left\{\tau_{0}>T\right\}}$ where $\tau_{0}$ is the first time a one dimensional Brownian motion leaves a bounded interval; the argument in [39] was based on a reduction to PDE whereas in the present work we will be working directly with the BSDE. To deal with the generality of the filtration, we impose a further technical assumption (see (C2), section 3 ): there exists a sequence $t_{n} \nearrow T$ such that the filtration $\mathbb{F}$ is left continuous at all $t_{n}$. See Remark 3.1 in Section 3 for comments on this assumption. To solve the BSDE with terminal condition $\xi_{2}$, we construct two sequences of processes (all solutions of the BSDE (1.5),(1.6) with different terminal conditions), one increasing and one decreasing such that the decreasing sequence dominates the increasing one. The limit of the increasing sequence is our candidate solution (in fact it is exactly the minimal supersolution of [23] with terminal condition $\xi_{2}$ ); the decreasing sequence is used to prove that the candidate solution satisfies the terminal condition. The terminal condition for the increasing sequence is $Y_{T}=n \cdot \mathbf{1}_{A_{T}}$ and for the decreasing sequence it is $Y_{T}=\infty \cdot \mathbf{1}_{A_{t_{n}}}$. That all these sequences are in the right order will be proved by the comparison principle for the BSDE (1.5) derived in [23].

An advantage of the results obtained in [39] is the following: they characterize precisely the minimal super-solution as the pasting of two process at a stopping time $\tau$. We have a partial result in that direction for $\xi_{1}$ : Proposition 2.6 proves this character of the minimal supersolution of the BSDE (1.5), (1.6) for the terminal condition $\xi_{1}$ when the filtration is assumed to be generated by $W$ and $\pi$. The proof of an analogous result for the terminal condition $\xi_{2}$ remains for future work.

Both [39] and the present work relax the assumption on $q>3$ imposed in [33, 35, 27] (see subsection 1.3 for further comments on these works). Parallel to the analysis in [39], the treatment of terminal conditions $\xi_{1}$ and $\xi_{2}$ in the present work involve different assumptions on $q$ : the proof of continuity of $Y$ given in Section 2 for the terminal condition $\xi_{1}$ requires $q>2$ whereas $q>1$ suffices for $\xi_{2}$ (Section 3). The reason for the difference is essentially the same as in [39]: for $\xi_{1}$ we construct an additional linear process for the upperbound; for $\xi_{2}$ the sequence of upperbounds are all solutions to the same BSDE with different terminal conditions. An additional difference in assumptions not present in [39]: an assumption adapted from the general framework of [23] introduces a constant $\ell>1$ (see (B2) below). In [39], this condition trivially holds and is not needed explicitly. In the treatment of $\xi_{1}$ we require $\ell>2$ (see Lemma 2.1); $\ell>1$ suffices for $\xi_{2}$. The reason for the difference is the same as for $q$ : in the treatment of $\xi_{1}$ we construct a linear BSDE as the upperbound and this requires more stringent integrability conditions on terminal values.

In Section 4 we identify a class of stopping times satisfying the assumptions made on the stopping times above. The class of these stopping times is defined in terms of a diffusion process $X$ driven by the Brownian motion $W$ :

$$
X_{t}=x_{0}+\int_{0}^{t} b\left(s, X_{s}\right) d s+\int_{0}^{t} \sigma\left(s, X_{s}\right) d W_{s},
$$

where $a=\sigma \sigma^{\prime}$ is assumed to be uniformly and strictly elliptic and $a$ and $b$ assumed uniformly Hölder continuous; these assumptions are adopted from [16, page 8]. The 
initial value $x_{0}$ takes values in a bounded open set $D_{0}$. Define

$$
D=\bigcup_{t=0}^{T}\{t\} \times D_{t} \subset \mathbb{R}^{d+1} ;
$$

$D$ satisfies the assumptions in [16], see Section 4 below. The class of stopping times identified in this section are exit times of $X$ from the domain $D$ :

$$
\tau \doteq \inf \left\{t \geq 0: X_{t} \in D_{t}^{c}\right\}
$$

To prove that $\tau$ satisfies the assumptions of Sections 2 and 3 it suffices to show that it has a continuous density. Despite the considerable literature on exit times of diffusions we are not aware of a result in the currently available literature establishing that the exit time $\tau$ of (1.9) has a density. Section 4 is devoted to the derivation of this density; the natural tool for this is the Green's function of the generator of $X$ derived in [16].

The rest of this introduction discusses the implications of our results to stochastic optimal control, lists the assumptions we adopt and the results we will be using from prior work and gives a summary of what is known in the prior literature about the continuity of the minimal supersolution of BSDE with singular terminal conditions. In Section 5 we comment on possible future work.

\subsection{Implications of continuity results for stochastic optimal control}

Minimal supersolutions of the BSDE (1.5) with singular terminal conditions can be used to represent the value function of a corresponding stochastic optimal control problem with constraints, see [1, 23] and [39, Section 4] for the precise formulation of the optimal control problem and a detailed discussion. In this connection between the BSDE and its corresponding stochastic optimal control problem, changing the terminal condition of the BSDE corresponds to changing the terminal payoff and the constraints of the problem. A natural question: when these change, do the value function and the optimal control of the control problem change? Surprisingly, and to the best of our knowledge, for the control problems corresponding to the class of terminal conditions treated in the present work, the current BSDE theory can't answer this question. The continuity results we prove in the present article establishing that a minimal supersolution is a solution in the sense of (1.2) provides an answer as follows. Suppose $Y^{(1)}$ and $Y^{(2)}$ are minimal supersolutions of the BSDE for two distinct terminal conditions $\xi^{(1)}, \xi^{(2)}$. Suppose that $Y^{(i)}$ are solutions to the BSDE with these terminal conditions in the sense of (1.2), i.e., that $Y^{(i)}$ are both continuous at time $T$. This and $\xi^{(1)} \neq \xi^{(2)}$ imply that $Y^{(1)}$ and $Y^{(2)}$ are distinct processes. To rephrase this in terms of the control interpretation: changing the constraint and terminal value of the control problem from $\xi^{(1)}$ to $\xi^{(2)}$ leads to distinct value functions (and hence optimal controls) for the control problem.

We explain a further implication of the continuity results to optimal control through the following example. Let $X$ denote the state process of the corresponding optimal control problem. As explained in [1, 23] the terminal condition $\xi^{(1)}=\infty$ corresponds to the constraint $X_{T}=0$. Let us relax this constraint to requiring $X_{T}=0$ only when $\{\tau>T\}$ where $\tau$ is a stopping time of the filtration. The corresponding terminal condition $\xi=\infty \cdot \mathbf{1}_{\{\tau>T\}}$ belongs to the class we treat in Section 3. Two questions: 1) does this relaxation lead to a lower value function? This question is a special case of the question discussed in the previous paragraph, i.e., whether the same BSDE with distinct terminal conditions have distinct solutions, and we know that continuity of the solution implies that the solutions will be distinct. A more delicate question: 2$)$ is the optimal control tight, i.e., is it the case that, under the optimal control $X_{T}=0$ if and only if $\{\tau<T\}$ ? 
The continuity of the minimal supersolution implies that the answer to this question is also affirmative. In finance applications a non-tight optimal control can be interpreted as a strictly super-hedging trading strategy. Continuity results overrule such strategies.

As a last point in connection with optimal control and optimal liquidation we note that the continuity of the minimal supersolution at terminal time appears in [3], as a condition for the solution of an optimal targeting problem.

\subsection{Assumptions and results from prior works}

First let us state assumptions and results from the prior literature concerning nonsingular terminal condition $\xi$, that is there exists $p>1$ such that

$$
\mathbb{E}|\xi|^{p}<+\infty
$$

Let us define $\mathbb{L}_{\mu}^{\rho}=\mathbb{L}^{\rho}(\mathcal{E}, \mu ; \mathbb{R})$, the set of measurable functions $\psi: \mathcal{E} \rightarrow \mathbb{R}$ such that

$$
\|\psi\|_{\mathbb{L}_{\mu}^{\rho}}^{\rho}=\int_{\mathcal{E}}|\psi(e)|^{\rho} \mu(d e)<+\infty, \quad \text { and } \quad \mathfrak{B}_{\mu}^{2}= \begin{cases}\mathbb{L}_{\mu}^{2} & \text { if } p \geq 2 \\ \mathbb{L}_{\mu}^{1}+\mathbb{L}_{\mu}^{2} & \text { if } p<2 .\end{cases}
$$

For the definition of the sum of two Banach spaces, see for example [21]. The introduction of $\mathfrak{B}_{\mu}^{2}$ is motivated in [24]. We assume that $f: \Omega \times[0, T] \times \mathbb{R} \times \mathbb{R}^{m} \times \mathfrak{B}_{\mu}^{2} \rightarrow \mathbb{R}$ is a random measurable function, such that for any $(y, z, \psi) \in \mathbb{R} \times \mathbb{R}^{m} \times \mathfrak{B}_{\mu}^{2}$, the process $f(t, y, z, \psi)$ is progressively measurable. For notational convenience we write $f_{t}^{0}=f(t, 0,0, \mathbf{0}), \mathbf{0}$ denotes the null application from $\mathbb{R}^{m}$ to $\mathbb{R}$.

The precise assumptions on the driver $f$, adapted from [23] are as follows:

(A1) The function $y \mapsto f(t, y, z, \psi)$ is continuous and monotone: there exists $\chi \in \mathbb{R}$ such that a.s. and for any $t \in[0, T]$ and $z \in \mathbb{R}^{m}$ and $\psi \in \mathfrak{B}_{\mu}^{2}$

$$
\left(f(t, y, z, \psi)-f\left(t, y^{\prime}, z, \psi\right)\right)\left(y-y^{\prime}\right) \leq \chi\left(y-y^{\prime}\right)^{2} .
$$

(A2) $\sup _{|y| \leq n}\left|f(t, y, 0,0)-f_{t}^{0}\right| \in L^{1}((0, T) \times \Omega)$ holds for every $n>0$.

(A3) There exists a progressively measurable process $\kappa=\kappa^{y, z, \psi, \phi}: \Omega \times \mathbb{R}_{+} \times \mathbb{R}^{m} \times \mathfrak{B}_{\mu}^{2} \rightarrow$ $\mathbb{R}$ such that

$$
f(t, y, z, \psi)-f(t, y, z, \phi) \leq \int_{\mathcal{E}}(\psi(e)-\phi(e)) \kappa_{t}^{y, z, \psi, \phi}(e) \mu(d e)
$$

with $\mathbb{P} \otimes L e b \otimes \mu$-a.e. for any $(y, z, \psi, \phi),-1 \leq \kappa_{t}^{y, z, \psi, \phi}(e)$ and $\left|\kappa_{t}^{y, \psi, \phi}(e)\right| \leq \vartheta(e)$ where $\vartheta$ belongs to the dual space of $\mathfrak{B}_{\mu}^{2}$, that is $\mathbb{L}_{\mu}^{2}$ or $\mathbb{L}_{\mu}^{\infty} \cap \mathbb{L}_{\mu}^{2}$.

(A4) There exists a constant $L_{f}$ such that a.s.

$$
\left|f(t, y, z, \psi)-f\left(t, y, z^{\prime}, \psi\right)\right| \leq L_{f}\left|z-z^{\prime}\right|
$$

for any $\left(t, y, z, z^{\prime}, \psi\right)$.

The set of conditions (A) guarantees the existence and uniqueness of the solution of the BSDE (1.5) and (1.6) if for some $p>1,(1.10)$ holds together with

$$
\mathbb{E}\left[|\xi|^{p}+\left(\int_{0}^{T}\left|f_{t}^{0}\right| d t\right)^{p}\right]<+\infty .
$$

(see $[22,24]$ and the references therein). 
A key tool for BSDEs is the comparison principle which ensures that if $\xi^{1} \leq \xi^{2}$ a.s., if we can compare the generators $f^{1} \leq f^{2}$ along one solution and if the drivers satisfy the conditions (A), then the solutions can be compared: a.s. $Y^{1} \leq Y^{2}$. See, e.g., [12, Section 3.2], [22, Proposition 4] or [30, Section 5.3.6].

A second set of assumptions are needed to control the growth of the process $Y$ when the terminal condition can take the value $+\infty$. These assumptions generalize the superlinearity of $y \mapsto y|y|^{q-1}$ in (1.3) and are adapted from [23]:

(B1) There exists a constant $q>1$ and a positive process $\eta$ such that for any $y \geq 0$

$$
f(t, y, z, \psi) \leq-\frac{y}{\eta_{t}}|y|^{q-1}+f(t, 0, z, \psi) .
$$

(B2) There exists some $\ell>1$ such that

$$
\mathbb{E} \int_{0}^{T}\left[\left(\eta_{s}\right)^{\ell(p-1)}\right] d s<+\infty
$$

where $p$ is the Hölder conjugate of $q$.

(B3) The parameter $\vartheta$ of (A3) satisfies: for any $\varpi>2$

$$
\int_{\mathcal{E}}|\vartheta(e)|^{\varpi} \mu(d e)<+\infty .
$$

(B4) We suppose that $f^{0}$ satisfies

$$
f_{t}^{0} \geq 0, t \in[0, T] \text { a.s., } \quad \mathbb{E} \int_{0}^{T}\left(f_{s}^{0}\right)^{\ell} d s<+\infty .
$$

where $\ell>1$ is the constant in assumption (B2).

(B5) The filtration is left-continuous at time $T$ (see the discussion in [35, Section 1.2] on this condition).

We further suppose that the generator $(t, y) \mapsto-y|y|^{q-1} / \eta_{t}$ satisfies the (A) assumptions, which means that $\eta$ satisfies:

$$
\mathbb{E} \int_{0}^{T} \frac{1}{\eta_{t}} d t<+\infty
$$

Remark 1.1 (On Assumption (B3)). In fact it is sufficient to assume that $\vartheta$ belongs to some $\mathbb{L}_{\mu}^{\rho}$ for $\rho$ large enough. But this generality leads to cumbersome conditions on $\ell$ and $q$ in Theorem 2.5.

Remark 1.2 (On Condition (B4)). The work [23] introduces an integrability assumption on $\left(f_{t}^{0}\right)^{-}=\max \left(-f_{t}^{0}, 0\right)$ and on $\left(f_{t}^{0}\right)^{+}$(see conditions A4 and A6 in [23]). Hence (B4) is stronger. The sign hypothesis could be relaxed at the expense of more technical considerations and presentation.

The case $\int_{0}^{T}\left(f_{s}^{0}\right)^{+} d s=+\infty$ (excluded by our assumption (B4)) is not an obstacle to the construction of minimal supersolutions, and [23], which constructs minimal supersolutions allows $\int_{0}^{T}\left(f_{s}^{0}\right)^{+} d s=+\infty$. The problem with $\int_{0}^{T}\left(f_{s}^{0}\right)^{+} d s=+\infty$ is that in its presence it is known that $Y^{\mathrm{min}}$ may be discontinuous at $T$ : see, [35, Section 3.1], for a BSDE that violates the integrability condition (B4) on $f^{0}$, whose minimal supersolution $Y^{\text {min }}$ explodes almost surely at time $T$ for all terminal values $\xi$. Therefore, the integrability condition (B4) is natural when one seeks continuity results for the class of BSDE treated in the present work. 
From [23, Theorem 1], under the setting of conditions (A) and (B), we know that there exists a process $(Y, Z, \psi, M)$ which is a minimal supersolution to the BSDE (1.5) with singular terminal condition $Y_{T}=\xi \geq 0$ in the sense that:

1. for all $t<T$ :

$$
\mathbb{E}\left(\sup _{s \in[0, t]}\left|Y_{s}\right|^{\ell}+\left(\int_{0}^{t}\left|Z_{s}\right|^{2} d s\right)^{\ell / 2}+\left(\int_{0}^{t} \int_{\mathcal{E}}\left|\psi_{s}(e)\right|^{2} \pi(d e, d s)\right)^{\ell / 2}+[M]_{t}^{\ell / 2}\right)<+\infty
$$

2. $Y$ is non-negative;

3. for all $0 \leq s \leq t<T$ :

$$
Y_{s}=Y_{t}+\int_{s}^{t} f\left(u, Y_{u}, Z_{u}, \psi_{u}\right) d u-\int_{s}^{t} Z_{s} d W_{s}-\int_{s}^{t} \int_{\mathcal{E}} \psi_{u}(e) \widetilde{\pi}(d e, d u)-\int_{s}^{t} d M_{u} .
$$

4. The terminal condition (1.6) becomes (1.1), namely: a.s.

$$
\liminf _{t \rightarrow T} Y_{s} \geq \xi
$$

5. For any other supersolution $\left(Y^{\prime}, Z^{\prime}, \psi^{\prime}, M^{\prime}\right)$ satisfying the first four properties, we have $Y_{t} \leq Y_{t}^{\prime}$ a.s. for any $t \in[0, T)$.

As in [39], we denote this minimal supersolution by $\left(Y^{\min }, Z^{\min }, \psi^{\min }, M^{\text {min }}\right)$. Let us recall that the construction is done by approximation ([23, Theorem 1]). We consider $\left(Y^{(k)}, Z^{(k)}, \psi^{(k)}, M^{(k)}\right)$ the unique solution of the BSDE:

$$
\begin{aligned}
Y_{t}^{(k)} & =\xi \wedge k+\int_{t}^{T} f^{k}\left(s, Y_{s}^{(k)}, Z_{s}^{(k)}, \psi_{s}^{(k)}\right) d s \\
& -\int_{t}^{T} Z_{s}^{(k)} d W_{s}-\int_{t}^{T} \int_{\mathcal{E}} \psi_{s}^{(k)}(e) \widetilde{\pi}(d e, d s)-\int_{t}^{T} d M_{s}^{(k)},
\end{aligned}
$$

with truncated parameters, namely the terminal condition $\xi \wedge k$ and the driver

$$
f^{k}(t, y, z, \psi)=\left[f(t, y, z, \psi)-f_{t}^{0}\right]+\left(f_{t}^{0} \wedge k\right) .
$$

Under (A) and (B4), existence and uniqueness of the solution is guaranteed by [22, Theorem 3]. From the comparison principle ([22, Proposition 4]), the sequence $Y^{(k)}$ is non-decreasing and converges to a limit $Y^{\mathrm{min}}$ : a.s. for any $t \in[0, T]$

$$
\lim _{k \rightarrow+\infty} Y_{t}^{(k)}=Y_{t}^{\min }
$$

The sequence $\left(Z^{(k)}, \psi^{(k)}, M^{(k)}\right)$ converges to $\left(Z^{\min }, \psi^{\min }, M^{\min }\right)$ : for any $0 \leq t<T$

$$
\begin{aligned}
& \lim _{k \rightarrow+\infty} \mathbb{E} {\left[\left(\int_{0}^{t}\left|Z_{u}^{(k)}-Z_{u}^{\min }\right|^{2} d u\right)^{\ell / 2}+\left(\int_{0}^{t} \int_{\mathcal{E}}\left|\psi_{u}^{(k)}(e)-\psi_{u}^{\min }(e)\right|^{2} \pi(d e, d u)\right)^{\ell / 2}\right.} \\
&\left.+\left(\left[M^{(k)}-M^{\min }\right]_{t}\right)^{\ell / 2}\right]=0 .
\end{aligned}
$$

See the proof of [23, Proposition 3].

Finally following the arguments of [23, Propositions 2 and 3], we can prove the a priori upper estimate on the supersolution: for any $1<\ell^{\prime} \leq \ell$,

$$
Y_{t}^{\min } \leq \frac{K_{\vartheta, L_{f}, \ell^{\prime}}}{(T-t)^{p-\frac{\ell-\ell^{\prime}}{\ell \ell^{\prime}}}}\left[\mathbb{E}\left(\int_{t}^{T}\left(\left((p-1) \eta_{s}\right)^{p-1}+(T-s)^{p}\left(f_{s}^{0}\right)\right)^{\ell} d s \mid \mathcal{F}_{t}\right)\right]^{1 / \ell}
$$

where $K_{\vartheta, L_{f}, \ell^{\prime}}$ is a constant depending only on $\vartheta, L_{f}$ and $\ell^{\prime}$. This estimate is valid for any terminal value $\xi$. The proof of this estimate is given in Appendix A. 


\subsection{Continuity results in the prior literature}

The present article addresses the following questions on the minimal supersolution $Y^{\mathrm{min}}$ of the BSDE (1.5) and the terminal conditions $\xi_{1}$ and $\xi_{2}$ :

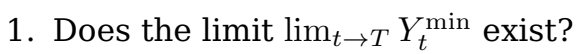

2. Can the inequality (1.1) be an equality (if the filtration is left-continuous at time $T$ ), i.e., is the supersolution $Y_{t}^{\mathrm{min}}$ in fact a solution?

Let us summarize the known results about these questions in the currently available literature. The existence of a limit at time $T$ is proved under a structural condition on the generator $f$ ([35, Theorem 3.1]) where the main idea is to show that $Y$ is a non-linear continuous transform of a non-negative supermartingale.

The second question is addressed in [33, 35, 39, 27]. In the first two papers [33, 35], the terminal condition $\xi$ is supposed to be Markovian ${ }^{2}$, that is $\xi=g\left(X_{T}\right)$, where $X$ is given by (1.8) ${ }^{3}$. In [27], $\xi$ is given by a smooth functional (in the sense of Dupire $[14,9,8])$ on the paths of $X$. In these three papers, the proof is based on the Itô formula and on a suitable control on $Z$ and $\psi$, and requires that the $q$ in (B1) is greater than 3 .

The work [39] treating the BSDE (1.3) and the terminal conditions (1.4) was a first attempt to obtain a positive answer to these questions with non-Markovian terminal conditions. This work obtains the continuity of $Y$ at time $T$ under the assumption $q>2$ for $\xi=\infty \cdot \mathbf{1}_{\left\{\tau_{0} \leq T\right\}}$ and $q>1$ for $\xi=\infty \cdot \mathbf{1}_{\{\tau>T\}}$. As we have already noted, the aim of the present work is to extend [39] in the directions indicated in the list following (1.3).

\section{Terminal condition $\xi_{1}$}

The goal of this section is to solve the BSDE (1.5) with terminal condition $\xi_{1}=$ $\infty \cdot \mathbf{1}_{\{\tau \leq T\}}$ where $\tau$ is any stopping time whose distribution in a neighborhood of $T$ has a bounded density. We will see in Section 4 below that first exit times from time varying domains of multidimensional diffusions driven by $W$ satisfy this condition. Another simple example is provided by jump times of compound Poisson processes, which are Erlang distributed and they evidently have densities.

Recall that we suppose that the set of conditions (A) and (B) hold. Let $Y^{(k)}$ be the solution of the BSDE (1.13) with terminal condition

$$
Y_{T}^{(k)}=\xi \wedge k=k \cdot \mathbf{1}_{\{\tau \leq T\}} .
$$

The minimal supersolution of (1.5), by definition, is

$$
Y_{t}^{\min }=\lim _{k \rightarrow \infty} Y_{t}^{(k)} .
$$

We will construct our solution by showing that $Y^{\mathrm{min}}$ is in fact a solution, i.e., it satisfies

$$
\lim _{t \rightarrow T} Y_{t}^{\min }=\xi_{1} .
$$

The results in [23] imply that (2.1) holds for $\xi_{1}=\infty$. Therefore, it suffices to show (2.1) over the event $\{\tau>T\}$ where the right side of (2.1) is 0 . We will do so by constructing a positive upperbound process $Y^{\infty, u}$ on $Y^{\text {min }}$ that converges to 0 over the same event. Let $Y^{\infty}$ be the minimal supersolution of (1.5) with terminal condition $Y_{T}=\infty$ (if $f(y)=-y|y|^{q-1}$, then $\left.Y_{t}^{\infty}=((q-1)(T-t))^{-\frac{1}{q-1}}\right)$. Define

$$
\xi_{1}^{(\tau)} \doteq \mathbf{1}_{\{\tau<T\}} Y_{\tau}^{\infty} .
$$

\footnotetext{
${ }^{2}$ No additional assumption is supposed on $f$, that is the setting is only half-Markovian.

${ }^{3} \mathrm{~A}$ jump component driven by the Poisson random measure could be added in the case.
} 
The upperbound process $Y^{\infty, u}$ is defined as the solution of the BSDE with the terminal value $\xi_{1}^{(\tau)}=Y_{\tau}^{\infty} \mathbf{1}_{\tau \leq T}$ at the random time $\tau \wedge T$ and the (linear in $y$ ) generator

$$
g(t, y, z, \psi)=\chi \cdot y+f(t, 0, z, \psi)
$$

where $\chi$ is the constant in (A1). For this to be well defined we need the following lemma: Lemma 2.1. If the distribution of $\tau$ in a neighborhood of $T$ has a bounded density and if $\ell>2$ of (B2) and $q>2+\frac{2}{\ell-2}$, then there exists some $\varrho>1$

$$
\mathbb{E}_{(x, t)}\left[\left(\xi_{1}^{(\tau)}\right)^{\varrho}\right]<\infty
$$

Proof. The assumptions (B2) and (B4) imply that

$$
M_{t} \doteq \mathbb{E}\left[\int_{0}^{T}\left(\left((p-1) \eta_{s}\right)^{p-1}+(T-s)^{p}\left(f_{s}^{0}\right)\right)^{\ell} d s \mid \mathcal{F}_{t}\right]
$$

is a well defined non-negative martingale. The hypotheses $\eta_{t}>0$ and $f_{t}^{0} \geq 0$ imply

$$
\int_{0}^{T}\left(\left((p-1) \eta_{s}\right)^{p-1}+(T-s)^{p}\left(f_{s}^{0}\right)\right)^{\ell} d s \geq \int_{t}^{T}\left(\left((p-1) \eta_{s}\right)^{p-1}+(T-s)^{p}\left(f_{s}^{0}\right)\right)^{\ell} d s .
$$

This and the a priori bound (1.15) on $Y^{\infty}$ imply for any $1<\varrho<\ell$

$$
\begin{aligned}
\mathbb{E}_{(x, t)}\left[\mathbf{1}_{\{\tau<T\}}\left(Y_{\tau}^{\infty}\right)^{\varrho}\right] & \leq \mathbb{E}_{(x, t)}\left[\mathbf{1}_{\{\tau<T\}} \frac{K_{\vartheta, L_{f}, \ell^{\prime}}^{\varrho}}{(T \wedge \tau-t)^{\hat{p} \varrho}} M_{\tau \wedge T}^{\frac{\varrho}{\ell}}\right] \\
& \leq K_{\vartheta, L_{f}, \ell^{\prime}}^{\varrho} \mathbb{E}_{(x, t)}\left[\mathbf{1}_{\{\tau<T\}} \frac{1}{(T \wedge \tau-t)^{\kappa}}\right]^{\frac{\ell-\varrho}{\ell}} \mathbb{E}_{(x, t)}\left[M_{\tau \wedge T}\right]^{\frac{\varrho}{\ell}}
\end{aligned}
$$

where

$$
\kappa=\frac{\hat{p} \varrho \ell}{\ell-\varrho}, \quad \hat{p}=p-\frac{\ell-\ell^{\prime}}{\ell \ell^{\prime}}
$$

and where we used the Hölder inequality since $\varrho<\ell$.

Note that to show our result, from (1.15) it suffices to show $\mathbb{E}_{(x, t)}\left[\mathbf{1}_{\{T-\delta<\tau<T\}} \times\right.$ $\left.\frac{1}{(T \wedge \tau-t)^{\kappa}}\right]<\infty$ for some $\delta>0$. We have assumed that the distribution of $\tau$ in a neighborhood of $T$ has a bounded density, which we will denote by $f^{\tau}(t, u)$. Then:

$$
\mathbb{E}_{(x, t)}\left[\mathbf{1}_{\{T-\delta<\tau<T\}} \frac{1}{(T \wedge \tau-t)^{\kappa}}\right]=\int_{T-\delta}^{T} \frac{1}{(u-t)^{\kappa}} f^{\tau}(t, u) d u,
$$

for some $\delta>0$. The boundedness of $f^{\tau}$ implies that we obtain the desired result if $\kappa<1$, that is if

$$
p<\frac{\ell-\ell^{\prime}}{\ell \ell^{\prime}}+\frac{\ell-\varrho}{\varrho \ell}
$$

The right side is maximal for $\ell^{\prime}=\varrho=1$. Recall that $p>1$. Hence we need that $\ell>2$ and if $q>2+\frac{2}{\ell-2}$, then $p<2 \frac{\ell-1}{\ell}$. We can find $\varrho>1$ and $\ell^{\prime}>1$ such that the desired inequality holds.

Remark 2.2. In [39], the coefficients are bounded, that is, we can take $\ell=+\infty$ and we get back the condition $q>2$. 
The driver $g$ satisfies all conditions (A). Moreover the terminal time $\tau \wedge T$ is bounded. Hence we apply [22, 24, Theorem 3] and ensure the existence and the uniqueness of the solution $\left(Y^{\infty, u}, Z^{\infty, u}, \psi^{\infty, u}, M^{\infty, u}\right)$ such that for any $t \in[0, T]$

$$
\begin{aligned}
\mathbb{E}\left[\left|Y_{t \wedge \tau}^{\infty, u}\right|^{\varrho}+\int_{0}^{\tau \wedge T}\left|Y_{s}^{\infty, u}\right|^{\varrho} d s+\left(\int_{0}^{\tau \wedge T}\left|Z_{s}^{\infty, u}\right|^{2} d s\right)^{\varrho / 2}\right. \\
\left.\quad+\left(\int_{0}^{\tau \wedge T} \int_{\mathcal{E}}\left|\psi_{s}^{\infty, u}(e)\right|^{2} \pi(d e, d s)\right)^{\varrho / 2}+\left[M^{\infty, u}\right]_{\tau \wedge T}^{\varrho / 2}\right]<+\infty
\end{aligned}
$$

Note that if $f^{0} \equiv 0$ and $f$ does not depend on $z$ and $\psi$, then

$$
Y_{t}^{\infty, u}=\mathbb{E}\left[e^{\chi(\tau-t)} Y_{\tau}^{\infty} \mathbf{1}_{\{\tau<T\}} \mid \mathcal{F}_{t}\right] .
$$

We next prove that $Y^{\infty, u}$ does serve as an upper bound on $Y^{(k)}$ :

Lemma 2.3. $Y^{(k)}$ admits the upper bound

$$
Y_{t}^{(k)} \leq Y_{t}^{\infty, u}
$$

a.s. on the random interval $\llbracket 0, \tau \wedge T \rrbracket$.

Proof. The minimal solution $Y^{\infty}$ is constructed by approximation and for any $n \geq k$, we have: $k \cdot \mathbf{1}_{\{\tau \leq T\}} \leq n$ a.s. By the comparison principle for BSDEs, a.s. for any $t \in[0, T]$ : $Y_{t}^{(k)} \leq Y_{t}^{\infty}$. Hence a.s.

$$
Y_{\tau \wedge T}^{(k)}=Y_{\tau}^{(k)} \mathbf{1}_{\tau \leq T} \leq Y_{\tau}^{\infty} \mathbf{1}_{\tau \leq T}
$$

Since $Y^{(k)}$ solves the BSDE (1.5) on the whole interval $[0, T]$, the stopped process $Y^{(k), \tau}=Y_{. \wedge \tau}^{(k)}$ solves the same BSDE on the random interval $\llbracket 0, \tau \wedge T \rrbracket$.

Now $Y^{\infty, u}$ is the solution of the BSDE with the terminal value $\xi_{\tau}^{(1)}=Y_{\tau}^{\infty} \mathbf{1}_{\tau \leq T}$ at the random time $\tau \wedge T$ and the generator

$$
g(t, y, z, \psi)=\chi y+f(t, 0, z, \psi)
$$

From the assumptions (A) on $f$, for any $y \geq 0$, we have

$$
f(t, y, z, \psi) \leq f(t, y, z, \psi)-f(t, 0, z, \psi)+f(t, 0, z, \psi) \leq \chi y+f(t, 0, z, \psi)=g(t, y, z, \psi)
$$

Note that $Y^{(k)}$ and $Y^{\infty}$ are non-negative. Hence we can compare the drivers and deduce the claimed result by the comparison principle.

We now prove that the upper bound process has the continuity property we need at terminal time $T$ :

Lemma 2.4. The upper bound process $Y^{\infty, u}$ satisfies:

$$
\lim _{t \rightarrow T} Y_{t}^{\infty, u}=0
$$

a.s. on $\{\tau>T\}$ 
Proof. Indeed for any $0 \leq t \leq s$ :

$$
\begin{aligned}
Y_{t \wedge \tau \wedge T}^{\infty, u} & =Y_{s \wedge \tau \wedge T}^{\infty, u}+\int_{t \wedge \tau \wedge T}^{s \wedge \tau \wedge T} g\left(r, Y_{r}^{\infty, u}, Z_{r}^{\infty, u}, \psi_{r}^{\infty, u}\right) d r \\
& -\int_{t \wedge \tau \wedge T}^{s \wedge \tau \wedge T} Z_{r}^{\infty, u} d W_{r}-\int_{t \wedge \tau \wedge T}^{s \wedge \tau \wedge T} \int_{\mathcal{E}} \psi_{r}^{\infty, u}(e) \widetilde{\pi}(d e, d r)-\int_{t \wedge \tau \wedge T}^{s \wedge \tau \wedge T} d M_{r}^{\infty, u} \\
& =Y_{s \wedge \tau \wedge T}^{\infty, u}+\int_{t \wedge \tau \wedge T}^{s \wedge \tau \wedge T} \chi Y_{r}^{\infty, u} d r+\int_{t \wedge \tau \wedge T}^{s \wedge \tau \wedge T} f_{r}^{0} d r-\int_{t \wedge \tau \wedge T}^{s \wedge \tau \wedge T} d M_{r}^{\infty, u} \\
& +\int_{t \wedge \tau \wedge T}^{s \wedge \tau \wedge T}\left[f\left(r, 0, Z_{r}^{\infty, u}, \psi_{r}^{\infty, u}\right)-f\left(r, 0,0, \psi_{r}^{\infty, u}\right)\right] d r-\int_{t \wedge \tau \wedge T}^{s \wedge \tau \wedge T} Z_{r}^{\infty, u} d W_{r} \\
& +\int_{t \wedge \tau \wedge T}^{s \wedge \tau \wedge T}\left[f\left(r, 0,0, \psi_{r}^{\infty, u}\right)-f_{r}^{0}\right] d r-\int_{t \wedge \tau \wedge T}^{s \wedge \tau \wedge T} \int_{\mathcal{E}} \psi_{r}^{\infty, u}(e) \widetilde{\pi}(d e, d r)
\end{aligned}
$$

Using (A4), we can write

$$
f\left(r, 0, Z_{r}^{\infty, u}, \psi_{r}^{\infty, u}\right)-f\left(r, 0,0, \psi_{r}^{\infty, u}\right)=\varkappa_{r}^{\infty, u} Z_{r}^{\infty, u}
$$

where the process $\varkappa^{\infty, u}$ is bounded by $L_{f}$ uniformly in $r$ and $\omega$. Using (A3) we have

$$
f\left(r, 0,0, \psi_{r}^{\infty, u}\right)-f_{r}^{0} \leq \int_{\mathcal{E}} \psi_{r}^{\infty, u}(e) \kappa_{r}^{0,0, \psi^{\infty, u}, 0}(e) \mu(d e) .
$$

From the comparison principle for BSDE and the explicit formula for the solution of a linear BSDE, we have an explicit upper bound on $Y^{\infty, u}$ :

$$
Y_{t}^{\infty, u} \leq \mathbb{E}\left[\mathcal{E}_{t, \tau \wedge T} Y_{\tau}^{\infty} \mathbf{1}_{\tau \leq T}+\int_{t}^{\tau \wedge T} \mathcal{E}_{t, s} f_{s}^{0} d s \mid \mathcal{F}_{t}\right]=\Gamma_{t},
$$

where for $t \leq s$

$$
\mathcal{E}_{t, s}=\exp \left(\chi(s-t)+\int_{t}^{s} \varkappa_{r}^{\infty, u} d W_{r}-\frac{1}{2} \int_{t}^{s}\left|\varkappa_{r}^{\infty, u}\right|^{2} d r\right) V_{t, s}^{\infty}
$$

and $V^{\infty}$ is the Doléans-Dade exponential:

$$
V_{t, s}^{\infty}=1+\int_{t}^{s} \int_{\mathcal{E}} V_{t, u-}^{\infty} \kappa_{u}^{0,0, \psi^{\infty, u}, 0}(e) \widetilde{\pi}(d e, d u) .
$$

From assumptions (B3) and (B4), and with the Hölder inequality, we obtain that

$$
\mathbb{E}\left[\int_{0}^{\tau \wedge T} \mathcal{E}_{t, s} f_{s}^{0} d s\right] \leq \mathbb{E}\left[\int_{0}^{\tau \wedge T}\left|\mathcal{E}_{t, s}\right|^{\ell^{*}} d s\right]^{\frac{1}{\ell^{*}}} \mathbb{E}\left[\int_{0}^{\tau \wedge T}\left(f_{s}^{0}\right)^{\ell} d s\right]^{\frac{1}{\ell}} \leq C \mathbb{E}\left[\int_{0}^{T}\left(f_{s}^{0}\right)^{\ell} d s\right]^{\frac{1}{\ell}}
$$

where $\ell^{*}$ is the Hölder conjugate of $\ell$. Our framework implies that $\mathcal{E}_{\text {., }}$ has moments of all orders (see [38]). Thus a.s.

$$
\lim _{t \rightarrow T} \mathbb{E}\left[\int_{t}^{\tau \wedge T} \mathcal{E}_{t, s} f_{s}^{0} d s \mid \mathcal{F}_{t}\right]=0
$$

Same arguments, together with the integrability property proved in Lemma 2.1, lead to

$$
\mathbb{E}\left[\mathcal{E}_{t, \tau \wedge T} Y_{\tau}^{\infty} \mathbf{1}_{\tau \leq T} \mid \mathcal{F}_{t}\right] \leq \mathbb{E}\left[\mathcal{E}_{t, \tau \wedge T}^{\varrho^{*}} \mid \mathcal{F}_{t}\right]^{\frac{1}{\varrho^{*}}} \mathbb{E}\left[\left(\xi_{1}^{(\tau)}\right)^{\varrho} \mid \mathcal{F}_{t}\right]^{\frac{1}{\varrho}}
$$


From the left-continuity of the filtration at time $T$, a martingale cannot have a jump at time $T$, hence

$$
\lim _{t \rightarrow T} \mathbb{E}\left[\left(\xi_{1}^{(\tau)}\right)^{\varrho} \mid \mathcal{F}_{t}\right]^{\frac{1}{\varrho}}=\xi_{1}^{(\tau)} .
$$

Therefore if $\tau>T$,

$$
0 \leq \lim _{t \rightarrow T} Y_{t}^{\infty, u} \leq \lim _{t \rightarrow T} \Gamma_{t}=0,
$$

which achieves the proof of the lemma.

Combining the lemmas above we have the main result of this section:

Theorem 2.5. Under conditions (A) and (B), if the distribution of the stopping time $\tau$ is given by a bounded density in a neighborhood of $T, \ell>2$ and $q>2+\frac{2}{\ell-2}$, then the minimal supersolution with terminal condition $\xi_{1}$ satisfies

$$
\lim _{t \rightarrow T} Y_{t}^{\min }=\xi_{1}
$$

almost surely.

Proof. As stated in the beginning of this section it suffices to prove (2.4) over the event $\{\tau>T\}$ where $\xi_{1}=0$. By our assumptions on the driver $f, Y^{(k)}$ is non-negative; this and Lemma 2.3 gives

$$
0 \leq Y_{t}^{(k)} \leq Y_{t}^{\infty, u} .
$$

On the other hand, by Lemma 2.4, the limit as $t \rightarrow T$ of the right side is 0 over the event $\{\tau>T\}$. These imply (2.4).

This result generalizes the continuity result [39, Theorem 2.1]. If the setting of this former result was less general, we were able to describe precisely the minimal supersolution, namely that it is obtained by pasting two processes at time $\tau$. The presence of the orthogonal martingale $M$ complicates this approach in the present setting, but if the filtration is assumed to be generated by $W$ and $\pi$ alone then the same technique can be used here as well. The details are as follows.

Let $Y^{1, \tau}$ be the solution of the BSDE (1.5) in the time interval $\llbracket 0, \tau \wedge T \rrbracket$ with terminal condition $\xi_{1}^{(\tau)}$ (again we can apply [22, 24, Theorem 3] as for $Y^{\infty, u}$ ). Following the idea of [39, Theorem 2.1], let us define

$$
\widehat{Y}_{t} \doteq \begin{cases}Y_{t}^{1, \tau}, & t \leq \tau \wedge T \\ Y_{t}^{\infty}, & \tau<t \leq T\end{cases}
$$

where we assume that $\tau$ is an $\mathbb{F}^{W}$ stopping time, that is it just depends on the paths of $W$, and is predictable (exit times of Section 4 are a particular case). The jump times of $Y_{t}^{1, \tau}$ and of $Y^{\infty}$ coincide with the jump times of the Poisson random measure or of the orthogonal martingale component. A consequence of the Meyer theorem (see [36, Chapter 3, Theorem 4]) implies that the jump times of $\pi$ are totally inaccessible, hence a.s. cannot be equal to $\tau$. However we cannot exclude that the orthogonal martingale may have a jump at time $\tau$. The second issue is the definition of the martingale part $(Z, \psi, M)$. For the first two components, we can easily paste them together

$$
\widehat{Z}_{t} \doteq\left\{\begin{array}{ll}
Z_{t}^{1, \tau}, & t \leq \tau \wedge T \\
Z_{t}^{\infty}, & \tau<t \leq T
\end{array}, \quad \widehat{\psi}_{t}(e) \doteq\left\{\begin{array}{ll}
\psi_{t}^{1, \tau}(e), & t \leq \tau \wedge T \\
\psi_{t}^{\infty}(e), & \tau<t \leq T
\end{array} .\right.\right.
$$


Since $\tau$ is predictable, these two processes are also predictable and the stochastic integrals

$$
\int_{0}^{\cdot} \widehat{Z}_{t} d W_{t}, \quad \int_{0}^{\cdot} \int_{\mathcal{E}} \widehat{\psi}_{t}(e) \widetilde{\pi}(d e, d t)
$$

are well-defined and are local martingales on $[0, T)$. Nonetheless if we define $\widehat{M}$ similarly, we cannot ensure that this process is still a local martingale. For the parts with $Z$ and $\psi$, the local martingale property is due to the representation as a stochastic integral. Based on these observations we provide the following result on the pasting method under the assumption that the filtration is generated by $W$ and $\pi$ alone; the approach in the proof of this proposition is the generalization of the approach used in [39].

Proposition 2.6. Assume that the filtration is generated by $W$ and $\pi$ and that $\tau$ is predictable ${ }^{4}$. Then $\widehat{Y}_{t}$ solves the BSDE (1.5) on $[0, T]$ with terminal condition $\widehat{Y}_{T}=\xi_{1}$ and satisfies the continuity property at time T. Moreover $\widehat{Y}=Y^{\mathrm{min}}$.

Proof. Since there is no additional martingale $M$ in the definition of $Y^{1, \tau}$ and $Y^{\infty}$ and since $\tau$ is predictable, the resulting process $\widehat{Y}$ is continuous at time $\tau$.

Now let us fix $s<t<T$. On the set $\{\tau \leq s\}, \widehat{Y}_{r}=Y_{r}^{\infty}$ for any $r \in[s, t]$. Therefore we have

$$
\widehat{Y}_{s}=\widehat{Y}_{t}+\int_{s}^{t} f\left(r, \widehat{Y}_{r}, Z_{r}^{\infty}, \psi_{r}^{\infty}\right) d r-\int_{s}^{t} Z_{r}^{\infty} d W_{r}-\int_{s}^{t} \int_{\mathcal{E}} \psi_{r}^{\infty}(e) \widetilde{\pi}(d e, d r) .
$$

The dynamics of $Y^{1, \tau}$ is given by:

$$
\begin{aligned}
Y_{s \wedge \tau \wedge T}^{1, \tau} & =Y_{t \wedge \tau \wedge T}^{1, \tau}+\int_{s \wedge \tau \wedge T}^{t \wedge \tau \wedge T} f\left(r, Y_{r}^{1, \tau}, Z_{r}^{1, \tau}, \psi_{r}^{1, \tau}\right) d r \\
& -\int_{s \wedge \tau \wedge T}^{t \wedge \tau \wedge T} Z_{r}^{1, \tau} d W_{r}-\int_{s \wedge \tau \wedge T}^{t \wedge \tau \wedge T} \int_{\mathcal{E}} \psi_{r}^{1, \tau}(e) \widetilde{\pi}(d e, d r) .
\end{aligned}
$$

It implies that for $\{\tau \geq t\}, \widehat{Y}$ has the required dynamics. Finally for $\{\tau \in(s, t)\}$, we have

$$
\begin{aligned}
Y_{s}^{1, \tau} & =Y_{\tau}^{1, \tau}+\int_{s}^{\tau} f\left(r, Y_{r}^{1, \tau}, Z_{r}^{1, \tau}, \psi_{r}^{1, \tau}\right) d r \\
& -\int_{s}^{\tau} Z_{r}^{1, \tau} d W_{r}-\int_{s}^{\tau} \int_{\mathcal{E}} \psi_{r}^{1, \tau}(e) \widetilde{\pi}(d e, d r)
\end{aligned}
$$

and

$$
Y_{\tau}^{\infty}=Y_{t}^{\infty}+\int_{\tau}^{t} f\left(r, Y_{r}^{\infty}, Z_{r}^{\infty}, \psi_{r}^{\infty}\right) d r-\int_{\tau}^{t} Z_{r}^{\infty} d W_{r}-\int_{\tau}^{t} \int_{\mathcal{E}} \psi_{r}^{\infty}(e) \widetilde{\pi}(d e, d r)
$$

By the continuity of $\widehat{Y}$ at time $\tau$, we get the desired dynamics also in this case.

Finally let us show that $\widehat{Y}$ is continuous at time $T$. On the set $\{\tau<T\}$, we have

$$
\lim _{t \rightarrow T} \widehat{Y}_{t}=\liminf _{t \rightarrow T} \widehat{Y}_{t}=\liminf _{t \rightarrow T} Y_{t}^{\infty}=+\infty .
$$

And on $\{\tau \geq T\}$,

$$
\lim _{t \rightarrow T} \widehat{Y}_{t}=\lim _{t \rightarrow T} Y_{t}^{1, \tau}=\xi_{1}^{(\tau)}=0 .
$$

We can conclude that $\widehat{Y}$ satisfies the BSDE (1.5) on $[0, T]$ with terminal condition $\widehat{Y}_{T}=\xi_{1}$ and is continuous at time $T$.

\footnotetext{
${ }^{4}$ It is sufficient to suppose that $\tau$ is accessible or that $\tau$ does not coincide with a jump time of the Poisson measure.
} 
From the minimality of $Y^{\text {min }}$, we have immediately that $Y_{t}^{\min } \leq \widehat{Y}_{t}$, a.s. for any $t \in[0, T]$. To obtain the converse inequality, let us define

$$
\widehat{Y}_{t}^{n} \doteq \begin{cases}Y_{t}^{1, \tau, n}, & t \leq \tau \wedge T \\ Y_{t}^{n}, & \tau<t \leq T\end{cases}
$$

where $Y^{n}$ (resp. $Y^{1, \tau, n}$ ) is the solution of the BSDE (1.5) on $[0, T]$ (resp. on $[0, \tau \wedge T]$ ) with terminal condition $n$ (resp. $Y_{\tau}^{n} \mathbf{1}_{\tau \leq T}$ ). Then we have that for any $k \geq n, \widehat{Y}^{n} \leq Y^{(k)} \leq Y^{\min }$. By construction of $Y^{\infty}, \widehat{Y}^{n}$ converges to $\widehat{Y}$. Therefore we conclude that $\widehat{Y}=Y^{\text {min }}$ and this achieves the proof of the Proposition.

\section{Terminal condition $\xi_{2}$}

The goal of this section is to prove the continuity of the minimal supersolution for the terminal condition

$$
\xi=\xi_{2}=\infty \cdot \mathbf{1}_{A_{T}},
$$

where $A_{t}$ is a decreasing sequence of events adapted to our filtration: for any $s \leq t$, $A_{t} \subset A_{s}$ and $A_{t} \in \mathcal{F}_{t}$. If $\tau_{0}$ is a stopping time, the set $A_{t}=\left\{\tau_{0}>t\right\}$ provides an example. We also assume that:

(C1) Equality (1.7) holds, that is the sequence is left continuous at time $T$ in probability:

$$
\mathbb{P}\left(\bigcap_{t<T} A_{t} \backslash A_{T}\right)=0 .
$$

(C2) There exists an increasing sequence $\left(t_{n}, n \in \mathbb{N}\right), t_{n}<T$ for all $n, \lim _{n \rightarrow+\infty} t_{n}=T$, and the filtration $\mathbb{F}$ is left continuous at time $t_{n}$ for any $n$. Recall that we already assume left continuity of $\mathbb{F}$ at time $T$.

If $A_{t}$ is defined as $A_{t}=\left\{\tau_{0}>t\right\}$ through a stopping time $\tau_{0}$, assumption (C1) is equivalent to: $\mathbb{P}\left(\tau_{0}=T\right)=0$. In particular if $\tau_{0}$ has a density this condition is satisfied. Therefore, as in the previous section, if $\tau_{0}$ is the jump time of an $\mathbb{F}$-adapted compound Poisson process, then it generates a sequence $A_{t}$ satisfying (C1). The same comment applies to the exit times whose densities are derived in the next section.

Remark 3.1 (On Condition (C2)). If the filtration $\mathbb{F}$ is quasi left-continuous, then (C2) holds for any sequence $t_{n}$. In particular our hypothesis is valid if $\mathbb{F}$ is generated by $W$ and $\pi$.

The notion of jumps for a filtration has been studied in [20] (see also [37, Section 2]). Let us note that we are not able to construct a counter example, that is a filtration such that (C2) does not hold.

If $\tau$ is a stopping time satisfying $\mathbb{P}(\tau=T)=0, A_{t}=\{\tau \geq t\}$ defines a sequence satisfying the conditions above. Conversely, any decreasing sequence $A_{t}$ can be associated with a stopping time via the following definition:

$$
\tau \doteq \inf \left\{t: \omega \in A_{t}^{c}\right\} .
$$

Lemma 3.2. $\tau$ is a stopping time of the filtration $\mathbb{F}$. If (1.7) holds, then $A_{T}=\{\tau \geq T\}$.

Proof. The definition of $\tau$ and the fact that $A_{s}^{c}$ is decreasing imply

$$
\{\tau \leq t\}=\bigcap_{n=1}^{\infty} A_{t+1 / n}^{c} \in \bigcap_{n=1}^{\infty} \mathcal{F}_{t+1 / n} .
$$


The right continuity of the filtration $\mathbb{F}$ implies $\{\tau \leq t\} \in \mathcal{F}_{t}$ i.e., $\tau$ is a stopping time.

Again the definition of $\tau$ and $A_{s}^{c} \searrow A_{T}^{c}$ as $s \searrow T$ imply $\{\tau<T\}=\bigcup_{n=1}^{\infty} A_{T-1 / n}^{c}$ and

$$
\{\tau<T\} \subset A_{T}^{c} .
$$

The continuity in probability at time $T$ of $\left(A_{t}, t \geq 0\right)$ implies

$$
\mathbb{P}\left(A_{T}^{c} \backslash\{\tau<T\}\right)=0,
$$

which completes the proof of the lemma.

Let us denote again by $Y^{\infty}$ the minimal solution of the BSDE (1.5) with terminal condition $+\infty$ and set

$$
\chi_{n} \doteq \mathbf{1}_{A_{t_{n}}} .
$$

Let us define $\mathcal{Y}^{n}$ as the solution of the BSDE over the interval $\left[t_{n}, T\right]$ with generator

$$
\widetilde{f}(s, y, z, u)=\left[\left(f\left(s,\left(1-\chi_{n}\right) y, z, u\right)-f_{s}^{0}\right]+\left(1-\chi_{n}\right) f_{s}^{0}\right.
$$

and terminal condition $\mathcal{Y}_{T}^{n}=0$ :

$$
\begin{aligned}
\mathcal{Y}_{t}^{n} & =\int_{t}^{T}\left[\left(f\left(s,\left(1-\chi_{n}\right) \mathcal{Y}_{s}^{n}, \mathcal{Z}_{s}^{n}, \mathcal{U}_{s}^{n}\right)-f_{s}^{0}\right] d s+\int_{t}^{T}\left(1-\chi_{n}\right) f_{r}^{0} d r\right. \\
& -\int_{t}^{T} \mathcal{Z}_{s}^{n} d W_{s}-\int_{t}^{T} \int_{\mathcal{E}} \mathcal{U}_{s}^{n} \widetilde{\pi}(d e, d s)-\int_{t}^{T} d \mathcal{M}_{s}^{n} .
\end{aligned}
$$

The driver $\tilde{f}$ satisfies all assumptions (A) and (B4) holds. From [22, 24, Theorem 2], there exists a unique solution to this BSDE satisfying

$$
\mathbb{E}\left[\sup _{t \in\left[t_{n}, T\right]}\left|\mathcal{Y}_{t}^{n}\right|^{\ell}\right] \leq \mathbb{E} \int_{t_{n}}^{T}\left|f_{r}^{0}\right|^{\ell} d r .
$$

Moreover by the comparison principle ([22, Proposition 4]), a.s. for all $t \in\left[t_{n}, T\right], \mathcal{Y}_{t}^{n} \geq 0$. Let us also remark that if $f^{0} \equiv 0$, then $\mathcal{Y}^{n} \equiv 0$.

Define $Y^{\infty, u, n}$ as the solution of the BSDE (1.5) on $\left[0, t_{n}\right]$ with terminal condition

$$
Y_{t_{n}, u, n}^{\infty, u}=\chi_{n} Y_{t_{n}}^{\infty}+\left(1-\chi_{n}\right) \mathcal{Y}_{t_{n}}^{n} .
$$

Note that from (1.15), this terminal condition is in $L^{\ell}(\Omega)$, hence the solution is welldefined on $\left[0, t_{n}\right]$. We extend $Y^{\infty, u, n}$ on the whole interval $[0, T]$ : for all $t_{n} \leq t \leq T$ :

$$
Y_{t}^{\infty, u, n}=\chi_{n} Y_{t}^{\infty}+\left(1-\chi_{n}\right) \mathcal{Y}_{t}^{n} .
$$

Lemma 3.3. The process $Y^{\infty, u, n}$ satisfies the dynamics of the BSDE (1.5) on the whole interval $[0, T]$. Moreover a.s.

$$
\lim _{t \rightarrow T} Y_{t}^{\infty, u, n}=\infty \cdot \mathbf{1}_{A_{t_{n}}} .
$$

Proof. By the definition of $Y^{\infty}$, for any $t_{n} \leq t<s<T$, we have

$$
Y_{t}^{\infty}=Y_{s}^{\infty}+\int_{t}^{s} f\left(r, Y_{r}^{\infty}, Z_{r}^{\infty}, \psi_{r}^{\infty}\right) d r-\int_{t}^{s} Z_{r}^{\infty} d W_{r}-\int_{t}^{s} \int_{\mathcal{E}} \psi_{r}^{\infty}(e) \widetilde{\pi}(d e, d r)-\int_{t}^{s} d M_{r}^{\infty},
$$


hence multiplying both sides by $\chi_{n}$, which is $\mathcal{F}_{t_{n}}$-measurable, we obtain

$$
\begin{aligned}
\chi_{n} Y_{t}^{\infty} & =\chi_{n} Y_{s}^{\infty}+\int_{t}^{s} \chi_{n} f\left(r, Y_{r}^{\infty}, Z_{r}^{\infty}, \psi_{r}^{\infty}\right) d r \\
& -\int_{t}^{s} \chi_{n} Z_{r}^{\infty} d W_{r}-\int_{t}^{s} \int_{\mathcal{E}} \chi_{n} \psi_{r}^{\infty}(e) \widetilde{\pi}(d e, d r)-\int_{t}^{s} d \chi_{n} M_{r}^{\infty} \\
& =\chi_{n} Y_{s}^{\infty}+\int_{t}^{s}\left[f\left(r, \chi_{n} Y_{r}^{\infty}, \chi_{n} Z_{r}^{\infty}, \chi_{n} \psi_{r}^{\infty}\right)-f_{r}^{0}\right] d r+\int_{t}^{s} \chi_{n} f_{r}^{0} d r \\
& -\int_{t}^{s} \chi_{n} Z_{r}^{\infty} d W_{r}-\int_{t}^{s} \int_{\mathcal{E}} \chi_{n} \psi_{r}^{\infty}(e) \widetilde{\pi}(d e, d r)-\int_{t}^{s} \chi_{n} d M_{r}^{\infty} .
\end{aligned}
$$

And from the definition of $\mathcal{Y}^{n}$, we have

$$
\begin{aligned}
\left(1-\chi_{n}\right) \mathcal{Y}_{t}^{n} & =\int_{t}^{T}\left(1-\chi_{n}\right)\left[\left(f\left(s,\left(1-\chi_{n}\right) \mathcal{Y}_{s}^{n}, \mathcal{Z}_{s}^{n}, \mathcal{U}_{s}^{n}\right)-f_{s}^{0}\right] d s+\int_{t}^{T}\left(1-\chi_{n}\right) f_{r}^{0} d r\right. \\
& -\int_{t}^{T}\left(1-\chi_{n}\right) \mathcal{Z}_{s}^{n} d W_{s}-\int_{t}^{T} \int_{\mathcal{E}}\left(1-\chi_{n}\right) \mathcal{U}_{s}^{n} \widetilde{\pi}(d e, d s)-\int_{t}^{T}\left(1-\chi_{n}\right) d \mathcal{M}_{s}^{n} \\
& =\int_{t}^{T}\left[\left(f\left(s,\left(1-\chi_{n}\right) \mathcal{Y}_{s}^{n},\left(1-\chi_{n}\right) \mathcal{Z}_{s}^{n},\left(1-\chi_{n}\right) \mathcal{U}_{s}^{n}\right)-f_{s}^{0}\right] d s+\int_{t}^{T}\left(1-\chi_{n}\right) f_{r}^{0} d r\right. \\
& -\int_{t}^{T}\left(1-\chi_{n}\right) \mathcal{Z}_{s}^{n} d W_{s}-\int_{t}^{T} \int_{\mathcal{E}}\left(1-\chi_{n}\right) \mathcal{U}_{s}^{n} \tilde{\pi}(d e, d s)-\int_{t}^{T}\left(1-\chi_{n}\right) d \mathcal{M}_{s}^{n}
\end{aligned}
$$

Thereby $Y^{\infty, u, n}$ satisfies the dynamics of the BSDE (1.5) on $\left[t_{n}, T\right)$. Recall that the solution of a BSDE may have a jump at some given time $t$ if and only if the martingale parts $\tilde{\pi}$ or $M$ have a jump at time $t$. Hence from our assumption (C2), $Y^{\infty, u, n}$ is continuous at time $t_{n}$ and we can define

$$
\begin{aligned}
Z_{t}^{\infty, u, n} & \doteq \begin{cases}Z_{t}^{\infty, u, n}, & t \leq t_{n}, \\
\chi_{n} Z_{t}^{\infty}+\left(1-\chi_{n}\right) \mathcal{Z}_{t}^{n} & t_{n}<t \leq T,\end{cases} \\
\psi_{t}^{\infty, u, n}(e) & \doteq \begin{cases}\psi_{t}^{\infty, u, n}(e), & t \leq t_{n} \\
\chi_{n} \psi_{t}^{\infty}(e)+\left(1-\chi_{n}\right) \mathcal{U}_{t}^{n}(e) & t_{n}<t \leq T,\end{cases}
\end{aligned}
$$

and

$$
M_{t}^{\infty, u, n} \doteq \begin{cases}M_{t}^{\infty, u, n}, & t<t_{n} \\ \chi_{n} M_{t}^{\infty}+\left(1-\chi_{n}\right) \mathcal{M}_{t}^{n} & t_{n} \leq t \leq T\end{cases}
$$

Then we have that the process $\left(Y^{\infty, u, n}, Z^{\infty, u, n}, \psi^{\infty, u, n}, M^{\infty, u, n}\right)$ satisfies the dynamics of the BSDE (1.5) on the whole interval $[0, T)$ and with the singular terminal value $\infty \cdot \mathbf{1}_{A_{t_{n}}}$ : a.s.

$$
\lim _{t \rightarrow T} Y_{t}^{\infty, u, n}=\infty \cdot \mathbf{1}_{A_{t_{n}}} .
$$

The only remaining issue concerns $M^{\infty, u, n}$ : it is not clear a priori that it is a martingale on $[0, T)$. However $\left(Y^{\infty, u, n}, Z^{\infty, u, n}, \psi^{\infty, u, n}, M^{\infty, u, n}\right)$ has the dynamics of the BSDE (1.5) on the interval $\left[0, t_{n+1}\right]$, with terminal condition $\zeta=Y_{t_{n+1}}^{\infty, u, n}=\chi_{n} Y_{t_{n+1}}^{\infty}+\left(1-\chi_{n}\right) \mathcal{Y}_{t_{n+1}}^{n}$. This terminal value belongs to $L^{\ell}(\Omega)$. Hence there exists a unique solution $(y, z, v, m)$ to the BSDE (1.5) with terminal condition $\zeta$. From uniqueness on $\left[t_{n}, t_{n+1}\right], y=\chi_{n} Y^{\infty}+$ $\left(1-\chi_{n}\right) \mathcal{Y}^{n}$ and $m=\chi_{n} M^{\infty}+\left(1-\chi_{n}\right) \mathcal{M}^{n}$ on this interval. And by uniqueness on $\left[0, t_{n}\right]$ for the BSDE with driver $f$ and terminal condition $y_{t_{n}}, y=Y^{\infty, u, n}$ and $m=M^{\infty, u, n}$ on $\left[0, t_{n}\right]$. Since the martingale $m$ has no jump at time $t_{n}$ (Hypothesis (C2)), we obtain that $M^{\infty, u, n}$ is a martingale on $\left[0, t_{n+1}\right]$ and thus on $[0, T)$. 
Fix $k>0$ and let $\left(Y^{(k)}, Z^{(k)}, U^{(k)}, M^{(k)}\right)$ denote the solution of the BSDE with the truncated terminal condition

$$
Y_{T}^{(k)}=\xi \wedge k=k \mathbf{1}_{A_{T}} .
$$

We have the following bound on $Y^{(k)}$ :

Lemma 3.4. A.s. for all $t \in[0, T], k$ and $n$

$$
0 \leq Y_{t}^{(k)} \leq Y_{t}^{u, \infty, n}
$$

Proof. Set

$$
\begin{aligned}
\partial Y_{s} & =Y_{s}^{u, \infty, n}-Y_{s}^{(k)}, \quad \partial Z_{s}=Z_{s}^{u, \infty, n}-Z_{s}^{(k)} \\
\partial \psi_{s}(e) & =\psi_{s}^{u, \infty, n}(e)-\psi_{s}^{(k)}(e), \quad \partial M_{s}=M_{s}^{u, \infty, n}-M_{s}^{(k)} .
\end{aligned}
$$

We have

$$
\begin{aligned}
& f\left(t, Y_{t}^{u, \infty, n}, Z_{t}^{u, \infty, n}, \psi_{t}^{u, \infty, n}\right)-f\left(t, Y_{t}^{(k)}, Z_{t}^{(k)}, \psi_{t}^{(k)}\right) \\
& \quad=-c_{t} \widehat{Y}_{t}+b_{t} \widehat{Z}_{t}+\left(f\left(t, Y_{t}^{(k)}, Z_{t}^{(k)}, \psi_{t}^{u, \infty, n}\right)-f\left(t, Y_{t}^{(k)}, Z_{t}^{(k)}, \psi_{t}^{(k)}\right)\right)
\end{aligned}
$$

with

$$
-c_{t}=\frac{f\left(t, Y_{t}^{u, \infty, n}, Z_{t}^{u, \infty, n}, \psi_{t}^{u, \infty, n}\right)-f\left(t, Y_{t}^{(k)}, Z_{t}^{u, \infty, n}, \psi_{t}^{u, \infty, n}\right)}{\partial Y_{t}} \mathbf{1}_{\partial Y_{t} \neq 0}
$$

and

$$
b_{t}=\frac{f\left(t, Y_{t}^{(k)}, Z_{t}^{u, \infty, n}, \psi_{t}^{u, \infty, n}\right)-f\left(t, Y_{t}^{(k)}, Z_{t}^{(k)}, \psi_{t}^{u, \infty, n}\right)}{\partial Z_{t}} \mathbf{1}_{\partial Z_{t} \neq 0} .
$$

By assumption (A1) $-c_{t} \leq \chi$ and by (A4), $\left|b_{t}\right| \leq L_{f}$. For every $t<T$ the process $(\partial Y, \partial Z, \partial U, \partial M)$ solves the BSDE

$$
\begin{aligned}
d \partial Y_{s} & =\left[c_{s} \partial Y_{s}-b_{s} \partial Z_{s}-\left(f_{s}^{0}-k\right)^{+}-\left(f\left(s, Y_{s}^{(k)}, Z_{s}^{(k)}, \psi_{s}^{u, \infty, n}\right)-f\left(s, Y_{s}^{(k)}, Z_{s}^{(k)}, \psi_{s}^{(k)}\right)\right)\right] d s \\
& +\partial Z_{s} d W_{s}+\int_{\mathcal{E}} \partial \psi_{s}(e) \widetilde{\pi}(d e, d s)+d \partial M_{s}
\end{aligned}
$$

on $[0, t]$ with terminal condition $\partial Y_{t}=Y_{t}^{u, \infty, n}-Y_{t}^{(k)}$. Moreover, by Assumption (A3)

$$
f\left(s, Y_{s}^{(k)}, Z_{s}^{(k)}, \psi_{s}^{u, \infty, n}\right)-f\left(s, Y_{s}^{(k)}, Z_{s}^{(k)}, \psi_{s}^{(k)}\right) \geq \int_{\mathcal{E}} \kappa_{s}^{k, u, \infty, n}(e) \partial \psi_{s}(e) \mu(d e)
$$

where $\kappa^{k, u, \infty, n}=\kappa^{Y^{(k)}, Z^{(k)}, \psi^{(k)}, \psi^{u, \infty, n}}$. From [22, Lemma 10], we have

$$
\partial Y_{s} \geq \mathbb{E}\left[\partial Y_{t} \Gamma_{s, t}+\int_{s}^{t} \Gamma_{s, u}\left(f_{u}^{0}-k\right)^{+} d u \mid \mathcal{F}_{s}\right]
$$

where $\Gamma_{s, t}=\exp \left(-\int_{s}^{t} c_{u} d u+\frac{1}{2} \int_{s}^{t}\left(b_{u}\right)^{2} d u-\int_{s}^{t} b_{u} d W_{u}\right) \zeta_{s, t}$ and $\zeta_{s, t}$ solves

$$
\zeta_{s, t}=1+\int_{s}^{t} \zeta_{s, u-} \int_{\mathcal{E}} \kappa_{u}^{k, u, \infty, n}(e) \widetilde{\pi}(d e, d u)
$$

Our assumption (A3) ensures that $\zeta$ is non-negative and together with (A1), (A4) and (B3), $\Gamma$ verifies for any $p \geq 1$

$$
\mathbb{E}\left[\left(\Gamma_{s, T}\right)^{p}\right]<+\infty .
$$


See the appendix in [38]. We have $Y_{t}^{(k)} \leq(1+T) k$ and hence $\partial Y_{t} \geq-(1+T) k$. Thus

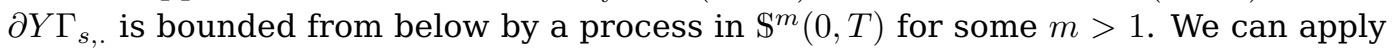
Fatou's lemma to obtain

$$
\partial Y_{s}=\liminf _{t \nearrow T} \mathbb{E}\left[\partial Y_{t} \Gamma_{s, t}+\int_{s}^{t} \Gamma_{s, u}\left(f_{u}^{0}-k\right)^{+} d u \mid \mathcal{F}_{s}\right] \geq \mathbb{E}\left[\liminf _{t \nearrow T}\left(\partial Y_{t} \Gamma_{s, t}\right) \mid \mathcal{F}_{s}\right] .
$$

The process $\left(\Gamma_{s, t}, s \leq t \leq T\right)$ is càdlàg and non-negative. Hence a.s.

$$
\liminf _{t \nearrow T}\left(\partial Y_{t} \Gamma_{s, t}\right)=\left(\liminf _{t \nearrow T} \partial Y_{t}\right) \Gamma_{s, T-} .
$$

But

$$
\liminf _{t \nearrow T} \partial Y_{t}=\infty \mathbf{1}_{A_{t_{n}}}-k \mathbf{1}_{A_{T}} \geq 0
$$

since $A_{T} \subset A_{t_{n}}$. This implies $Y_{s}^{u, \infty, n} \geq Y_{s}^{(k)}$ for any $s \in[0, T]$ and $k \geq 0$.

We now finish the proof of continuity of $Y$ at time $T$ :

Theorem 3.5. Under conditions (A), (B) and (C), the minimal supersolution with terminal condition $\xi_{2}$ satisfies

$$
\lim _{t \rightarrow T} Y_{t}^{\min }=\xi_{2}
$$

almost surely.

Proof. We know now that a.s.

$$
0 \leq Y_{t}^{(k)} \leq Y_{t}^{\min } \leq Y_{t}^{u, \infty, n}
$$

and we want to prove that for a.e. $\omega \in A_{T}^{c}$,

$$
\lim _{t \rightarrow T} Y_{t}^{\min }=0 .
$$

Recall that

$$
\mathbb{P}\left(\bigcap_{t<T} A_{t} \backslash A_{T}\right)=0
$$

Let us fix $\omega \in A_{T}^{c}$. We can assume (with probability 1) that $\omega$ belongs to $\bigcup_{t<T} A_{t}^{c}$, that is there exists $n$ such that $\omega \in A_{t_{n}}^{c}$. This implies:

$$
\limsup _{t \rightarrow T} Y_{t}^{\min }(\omega) \leq Y_{T}^{u, \infty, n}(\omega)=0
$$

\section{Density formula in terms of Green's function}

As noted in the introduction, one of the key ingredients in [39] in the analysis of the terminal condition $\mathbf{1}_{\left\{\tau_{0}<T\right\}}$ was the explicit formula available for the density of $\tau_{0}$, the first exit time of the Brownian motion from an interval $(a, b)$. The natural framework for the generalization of this formula to higher dimensions is the duality between Potential theory, elliptic / parabolic PDE and Diffusion processes [13]. Within this duality the exit times and the distribution of the path of the process up to the exit time corresponds to Green's functions [29]. The paper [11] uses the connection between hitting times and Green's functions to prove that the exit time of a one dimensional diffusion from a region has a density. A similar one dimensional computation is also given in [32]. Although the term "Green's function" doesn't appear in them, the works $[19,28]$ compute the Green's function for the Brownian motion in rectangular domains using the method of images; the work [5] extends this to three dimensions. The work [31], represents 
the distribution of the exit time of a $d$-dimensional diffusion from a fixed domain as the solution of a parabolic PDE. It identifies a smooth solution to the PDE whose derivative gives the density of the stopping time. The solution of the same PDE can be expressed in terms of the Green's function derived in the classical PDE book [16] by Friedman for the underlying parabolic PDE. The same Green's function can be used to prove that exit times of diffusions from domains that vary over time have densities. Given the duality between Green's functions and exit times, this is a natural result. But we have not been able to identify a reference in the current literature stating and proving it and therefore give its details in the present work.

The time variable in [16] corresponds to the time to maturity in the present setup. We state all definitions and results from [16] in terms of the time variable adopted in the present work (which is the one commonly used in the the stochastic processes framework); therefore, for example, the initial condition of [16] becomes the terminal condition and $t$ derivatives are multiplied by -.

Let $\mathcal{L}$ denote the parabolic operator associated with $X$ :

$$
\mathcal{L} u \doteq\langle\sigma(x, t), \sigma(x, t) H u\rangle+\left\langle b(x, t), \nabla_{x} u\right\rangle+\frac{\partial u}{\partial t},
$$

where $H u$ is the Hessian matrix of second derivatives of $u$. if we define

$$
a=\sigma \sigma^{\prime}
$$

the first term can also be written as $\langle a, H u\rangle$. To be able to use the results in [16] we adopt all of the assumptions it makes on $a$ and $b$, these are listed on [16, page 8]: $a$ is uniformly elliptic; $a$ and $b$ are Hölder continuous. The formal definition of Green's function is as follows ([16, page 82]):

Definition 4.1. A function $G(x, t, y, s)$ defined and continuous for $(x, t, y, s) \in \bar{D} \times(D \cup B)$, $t<s$ is called a Green's function of $\mathcal{L} u=0$ in $D$ if for any $0 \leq s \leq T$ and for any continuous function $f$ on $D_{s}$ having a compact support the function

$$
u(x, t)=\int_{D_{s}} G(x, t, y, s) f(y) d y
$$

is a solution of $L u=0$ in $D \cap\{0 \leq t<s\}$ and it satisfies the terminal and boundary conditions

$$
\begin{aligned}
\lim _{t \rightarrow s} u(x, t) & =f(x), x \in \bar{D}_{s} \\
u(x, t) & =0,(x, t) \in S \cap\{0 \leq t<s\} .
\end{aligned}
$$

The main result claiming the existence of Green's functions associated with $X$ is [16, Theorem 16, page 82]. This result is based on the following assumptions on the domain $D$ (listed as conditions $\bar{E}$ and $\overline{\bar{E}}$ on [16, pages 64,65]):

Assumption 4.2. For every point $(x, t) \in \bar{S}$ there exists an $(n+1)$-dimensional neighborhood $V$ such that $V \cap \bar{S}$ can be represented in the form

$$
x_{i}=h\left(x_{1}, \ldots, x_{i-1}, x_{i+1}, \ldots, x_{n}, t\right)
$$

for some $i \in\{1,2,3, \ldots, n\}, h, D_{x} h, D_{x}^{2} h$ and $D_{t} h$ exist and are Hölder continuous (exponent $\alpha$ ); $D_{x} D_{t} h, D_{t}^{2} h$ exist and are continuous.

The Green's function $G$ allows one to compute not just the distribution of the exit time of $X$ from a fixed domain but from a domain varying in time such as $D$; in fact it allows one to compute expectations of the form $\mathbb{E}_{(x, t)}\left[g\left(X_{s}\right) 1_{\{\tau>s\}}\right], s>t$. 
Proposition 4.3. Suppose $G$ is the Green's function of the operator $\mathcal{L}$. Then

$$
\mathbb{E}_{(x, t)}\left[g\left(X_{s}\right) 1_{\{\tau>s\}}\right]=\int_{D_{s}} g(y) G(x, t, y, s) d y,
$$

for any bounded continuous function $g$.

Proof. If $g$ has compact support in $D_{s}$, we know by the definition of $G$ that

$$
u(x, t)=\int_{D_{s}} g(y) G(x, t, y, s) d y,
$$

is a smooth solution of $\mathcal{L} u=0$ that is continuous in $\left.\bar{D}\right|_{[0, s]}$ with $u=0$ on $S$ and $u=g$ on $D_{s}$. Itô's formula applied to $u\left(X_{t}, t\right)$ gives (4.1). Thus it only remains to treat the case when $g$ doesn't have compact support in $D_{s}$. Let $g_{n}$ be a sequence of continuous functions with compact support in $D_{s}$ converging up to $g$. Then

$$
\mathbb{E}\left[g\left(X_{s}\right) 1_{\{\tau>s\}}\right]=\lim _{n \rightarrow \infty} \mathbb{E}\left[g_{n}\left(X_{s}\right) 1_{\{\tau>s\}}\right]+\mathbb{E}\left[g\left(X_{s}\right) 1_{\{\tau>s\}} \mathbf{1}_{\partial D_{s}}\left(X_{s}\right)\right] .
$$

The assumptions made on $a$ and $b$ imply that $X_{s}$ has a density in $\mathbb{R}^{n}$ and in particular the second expectation above is 0 . Therefore:

$$
\begin{aligned}
\mathbb{E}\left[g\left(X_{s}\right) 1_{\{\tau>s\}}\right] & =\lim _{n \rightarrow \infty} \mathbb{E}\left[g_{n}\left(X_{s}\right) 1_{\{\tau>s\}}\right] \\
& =\lim _{n \rightarrow \infty} \int_{D_{s}} g_{n}(y) G(x, t, y, s) d y=\int_{D_{s}} g(y) G(x, t, y, s) d y,
\end{aligned}
$$

where the last equality follows from the bounded convergence theorem.

Setting $g=1$ in (4.1) we get the following formula for $\mathbb{P}_{(x, t)}(\tau>s)$ :

$$
\mathbb{P}_{(x, t)}(\tau>s)=\int_{B_{T}} G(x, t, y, s) d y
$$

The density of the exit time $\tau$ is then

$$
-\frac{\partial}{\partial s} \int_{D_{s}} G(x, t, y, s) d y
$$

whenever this derivative exists. When the domain $D_{t}$ is constant, i.e., when $D_{t}=D_{0}$ for all $t$, the above derivative is simply

$$
-\frac{\partial}{\partial s} \int_{D_{0}} G(x, t, y, s) d y,=-\int_{D_{s}} G_{s}(x, t, y, s) d y=-\int_{D_{0}} G_{s}(x, t, y, s) d y,
$$

whenever $G_{s}$ exists and is continuous (by differentiation under the integral sign, see, e.g. [2]). Its computation in the presence of a time dependent domain $D_{t}$ is known as the Leibniz formula or the "Reynolds Transport Theorem" [15, 10]. All of the statements of this formula we have come across in the literature assume that the domain $D_{t}$ is given as the image of a smooth flow $\mathbf{x}(\cdot, t): D_{0} \mapsto D_{t}$. Assume for now $D_{t}$ can be represented as the image of $D_{0}$ under a smooth flow $\mathbf{x}$ and let $v$ denote the vector field defined by the flow (see the paragraph following Lemma 4.4 below for comments on the flow representation of $\left.D_{t}\right)$. Leibniz formula given in $[15,10]$ implies:

$$
-\frac{\partial}{\partial s} \int_{D_{s}} G(x, t, y, s) d y,=\int_{D_{s}} G_{s}(x, t, y, s) d y+\int_{\partial D_{s}} G(x, t, y, s)\langle v, N\rangle d S,
$$

where $N$ is the unit vector field on $\partial D_{s}$. A comparison of this with (4.3) shows that the second term in (4.4) is the additional term arising from the fact that $D_{t}$ varies in time. 
But by its construction the Green's function $G$ is 0 on $\partial D$ ([16, Corollary 1, page 83]), therefore this additional term is in fact 0 ! Then in the computation of the density of $\tau$, allowing the domain to vary in time doesn't have a direct impact on the density formula, (i.e, the formula (4.3) works both for time dependent domains as well as those that are independent of time).

Second observation about (4.4): for the derivative (4.2) to exist we need the partial derivative of $G$ with respect to $s$. We know by [16, Theorem 16, page 82] that $G$ is differentiable in its $t$ and $x$ variables. But this result does not directly address the smoothness of $G$ in the $s$ variable. One way to get smoothness of $G$ in the $s$ variable is to work with the Green's function $G^{*}$ of the adjoint operator $\mathcal{L}^{*}$ defined as follows:

$$
\mathcal{L}^{*} u \doteq\langle a, H u\rangle+\left\langle b^{*}, \nabla_{x} u\right\rangle+c^{*} u-\frac{\partial u}{\partial t}=0,
$$

where

$$
b_{i}^{*}=-b_{i}+2 \sum_{j=1}^{n} \frac{\partial a_{i, j}}{\partial x_{j}}, c^{*}=-\sum_{i=1}^{n} \frac{\partial b_{i}}{\partial x_{i}}+\sum_{i, j=1}^{n} \frac{\partial^{2} a_{i, j}}{\partial x_{i} \partial x_{j}} .
$$

For $G^{*}$ to exist and be smooth in its $x$ and $t$ variables it suffices that $b^{*}$ and $c^{*}$ be uniformly Hölder continuous (the uniform ellipticity of $a$ is already assumed).

Lemma 4.4. Let $b_{i}^{*}$ and $c^{*}$ of (4.5) be uniformly Hölder continuous. Then $G$ is differentiable in $s$ with a continuous derivative $G_{s}$.

Proof. The assumptions on $b_{i}^{*}$ and $c^{*}$ imply that the adjoint operator $\mathcal{L}^{*}$ satisfies the conditions of [16, Theorem 16, page 82] which says that $\mathcal{L}^{*}$ has associated with it a Green's function $G^{*}$ that is differentiable in $t$ with a continuous derivative $G_{t}^{*}$. By [16, Theorem 17, page 84] $G$ and $G^{*}$ are dual, i.e.,

$$
G(x, t, y, s)=G^{*}(y, s, x, t) ;
$$

this and the $G_{s}=G_{t}^{*}$ imply the statement of the lemma.

Even though in the end it has no influence on the final expression of the density, we need the existence of a continuously differentiable flow $\mathbf{x}$ that generates the domain $D$ to 1) invoke Leibniz rule and 2) to show that the resulting density is continuous. Many papers working on PDE with time dependent domains use this assumption $[7,6,10]$. Friedman's classical book [16] on parabolic PDE, on which most of the arguments above are based, does not contain this assumption directly. However, the assumptions already made on $D$ do indeed imply that $D_{t}$ can be represented as the forward image of $D_{0}$ under a smooth flow $\mathbf{x}$. To find such a flow one can proceed as follows: first use the local graph representation of $\partial D$ given in Assumption 4.2 to define a flow on $\partial D$ as follows:

$$
\mathbf{x}(x, t)=\left(h\left(x_{2}, x_{3}, \ldots, x_{d}, t\right), x_{2}, x_{3}, \ldots, x_{d}, t\right),
$$

where this definition is made in a neighborhood of $\left(x_{0}, t_{0}\right) \in \partial D$ where the graph of $h$ represents a portion of $\partial D$. That $h$ is $C^{1}$ implies that $\mathbf{x}$ defined as above is a smooth flow on $\partial D$. One can now extend this flow to all of $\mathbb{R}^{d}$ using classical results on the possibility of such an extension (see e.g., [6, page 584] or [25, page 201, Extension lemma for vector fields on submanifolds]). That $D_{t}$ is the forward image of $D_{0}$ now follows from the fact that $\mathbf{x}$, by its definition, leaves $\partial D$ invariant and the existence uniqueness theorem for ODE.

We can now make a precise statement about the density of $\tau$ : 
Proposition 4.5. Suppose $a$ is uniformly elliptic and $a, b, b^{*}$ and $c^{*}$ are uniformly Hölder continuous. and let $D$ satisfy the assumptions 4.2. Then the Green's function $G$ is continuously differentiable in $s$ and the exit time $\tau$ has continuous density

$$
f^{\tau}(x, t, s)=-\int_{D_{s}} G_{s}(x, t, y, s) d y, s \in(t, T] .
$$

Proof. The existence and continuity of $G_{s}$ follows from Lemma 4.4; the density formula follows from Leibniz's rule and $G=0$ on $\partial D_{t}$, as discussed above. The continuity of the density follows from the continuity of $G_{s}$ and the fact that $D_{t}$ is the smooth image of $D_{0}$ under the flow $\mathbf{x}$.

\section{Conclusion}

The present work finds solutions to BSDE (1.5) with a superlinear driver with singular terminal values of the form $\mathbf{1}_{A}, A \in \mathcal{F}_{T}$. In studying this question it generalizes the class of events $A$, the assumptions on the driver $f$ as well as the filtration $\mathcal{F}_{T}$ as compared to the previous work [39], which focused on a deterministic $f$, the filtration generated by a Brownian motion and $A$ of the form $\left\{\tau_{0} \leq T\right\}$ and $\left\{\tau_{0}>T\right\}$ where $\tau_{0}$ is the first exit time of the Brownian motion from a fixed interval. With the results of Section 3 we see that under general conditions on the driver and the filtration, the BSDE (1.5) with terminal condition $\mathbf{1}_{A} \cdot \infty$ can be solved for any $A \in \mathcal{F}_{T}$ that can be written as the limit of a decreasing sequence of adapted events. The arguments in Section2 imply that for events the form $\{\tau \leq T\}$, where $\tau$ is a stopping time to obtain continuous solutions to the BSDE we only need that $\tau$ has a bounded density. In Section 4 we show that exit times of multidimensional Markovian diffusions from time dependent smooth domains satisfy this condition. The identification of all events $A$ in $\mathcal{F}_{T}$ for which the BSDE (1.5) with terminal condition $\infty \cdot \mathbf{1}_{A}$ has a continuous solution remains an open problem. As already noted we rely on the density of $\tau$ in dealing with the event $A=\{\tau \leq T\}$; this reliance brings with it the assumption $q>2$ when dealing with the terminal condition $\mathbf{1}_{A} \cdot \infty$. To remove this assumption is an open problem for future research.

Another natural direction for future research is the derivation of density formulas for exit times for more general multidimensional processes, including those with jumps. Once such formulas are available the arguments in Section 2 would imply the existence of solution to BSDE (1.5) with terminal conditions defined by these exit times.

All results obtained in this paper can be generalized to the case where the compensator of $\pi$ is random and equivalent to the measure $\mu \otimes d t$ with a bounded density for example (see the introduction of [4] for example). Nevertheless since we refer to [24, 22] for the existence and uniqueness of the solution of BSDE, we keep this setting for $\pi$.

\section{A Proof of the upper bound (1.15)}

Let us recall the arguments of the proof of [23, Proposition 2]. For any $k \geq 0$ we consider the BSDE (1.13)

$$
d Y_{t}^{(k)}=-f^{k}\left(t, Y_{t}^{(k)}, Z_{t}^{(k)}, \psi_{t}^{(k)}\right) d t+Z_{t}^{(k)} d W_{t}+\int_{\mathcal{E}} \psi_{t}^{(k)}(e) \widetilde{\pi}(d e, d t)+d M_{t}^{(k)}
$$

with bounded terminal condition $Y_{T}^{(k)}=\xi \wedge k$ and where $f^{k}$ is given by (1.14):

$$
f^{k}(t, y, z, \psi)=\left(f(t, y, z, \psi)-f_{t}^{0}\right)+f_{t}^{0} \wedge k .
$$

The solution $Y^{(k)}$ is non-negative in our setting. We also consider the driver

$$
h(t, y, z, \psi)=b_{t}^{k}-p \frac{1}{T-t} y+\left[f(t, 0, z, \psi)-f_{t}^{0}\right] .
$$


with $b_{t}^{k}=\frac{\left((p-1) \eta_{t}\right)^{p}}{(T-t)^{p}}+\left(f_{t}^{0} \wedge k\right)$. Let $\varepsilon>0$ and denote by $\left(\mathcal{Y}^{\varepsilon, k}, Z^{\varepsilon, k}, \phi^{\varepsilon, k}, N^{\varepsilon, k}\right)$ the solution process of the BSDE on $[0, T-\varepsilon]$ with driver $h$ and terminal condition $\mathcal{Y}_{T-\varepsilon}^{\varepsilon, k}=Y_{T-\varepsilon}^{(k)} \geq 0$. Recall that from (A3) and (A4)

$$
f(t, 0, z, \psi)-f_{t}^{0} \leq \beta_{t}^{z, \psi} z+\int_{\mathcal{E}} \psi(e) \kappa_{t}^{0,0, \psi, 0}(e) \mu(d e),
$$

where

$$
\beta_{t}^{z, \psi}=\frac{f(t, 0, z, \psi)-f(t, 0,0, \psi)}{z \mathbf{1}_{z \neq 0}} .
$$

From (A4), $\beta^{z, \psi}$ is a bounded process by $L_{f}$. Hence by a comparison argument with the solution for linear BSDE (see [38, Lemma 4.1]) we have

$$
\mathcal{Y}_{t}^{\varepsilon, k} \leq \mathbb{E}\left[\Gamma_{t, T-\varepsilon} Y_{T-\varepsilon}^{(k)}+\int_{t}^{T-\varepsilon} \Gamma_{t, s} b_{s}^{k} d s \mid \mathcal{F}_{t}\right]
$$

where for $t \leq s \leq T-\varepsilon$

$$
\begin{aligned}
\Gamma_{t, s} & =\exp \left(-\int_{t}^{s} \frac{p}{T-u} d u+\int_{t}^{s} \beta_{u}^{Z^{\varepsilon, k}, \phi^{\varepsilon, k}} d W_{u}-\frac{1}{2} \int_{t}^{s}\left(\beta_{u}^{Z^{\varepsilon, k}, \phi^{\varepsilon, k}}\right)^{2} d u\right) V_{t, s}^{\varepsilon, k} \\
& =\left(\frac{T-s}{T-t}\right)^{p} \exp \left(\int_{t}^{s} \beta_{u}^{Z^{\varepsilon, k}, \phi^{\varepsilon, k}} d W_{u}-\frac{1}{2} \int_{t}^{s}\left(\beta_{u}^{Z^{\varepsilon, k}, \phi^{\varepsilon, k}}\right)^{2} d u\right) V_{t, s}^{\varepsilon, k}
\end{aligned}
$$

and

$$
V_{t, s}^{\varepsilon, k}=1+\int_{t}^{s} \int_{\mathcal{Z}} V_{t, u^{-}}^{\varepsilon, k} \kappa_{u}^{0, \phi^{\varepsilon, k}, 0}(z) \widetilde{\pi}(d z, d u)
$$

Hence

$$
\mathcal{Y}_{t}^{\varepsilon, k} \leq \frac{1}{(T-t)^{p}} \mathbb{E}\left[\varepsilon^{\rho} V_{t, T-\varepsilon}^{\varepsilon, k} Y_{T-\varepsilon}^{(k)}+\int_{t}^{T-\varepsilon} V_{t, s}^{\varepsilon, k}(T-s)^{p} b_{s}^{k} d s \mid \mathcal{F}_{t}\right]
$$

Since $b^{k} \geq 0$ it holds that $\mathcal{Y}_{t}^{\varepsilon, k} \geq 0$ a.s. for every $t \in[0, T]$. Hence from Condition (B1)

$$
f^{k}\left(t, \mathcal{Y}_{t}^{\varepsilon, k}, Z_{t}^{\varepsilon, k}, \phi_{t}^{\varepsilon, k}\right) \leq-\frac{1}{\eta_{t}}\left(\mathcal{Y}_{t}^{\varepsilon, k}\right)^{q}+f^{k}\left(t, 0, Z_{t}^{\varepsilon, k}, \phi_{t}^{\varepsilon, k}\right)
$$

It follows that

$$
\begin{aligned}
f^{k}\left(t, \mathcal{Y}_{t}^{\varepsilon, k}, Z_{t}^{\varepsilon, k}, \phi_{t}^{\varepsilon, k}\right) & \leq h\left(t, \mathcal{Y}_{t}^{\varepsilon, k}, Z_{t}^{\varepsilon, k}, \phi_{t}^{\varepsilon, k}\right)-\frac{1}{\eta_{t}}\left(\mathcal{Y}_{t}^{\varepsilon, k}\right)^{q}-\frac{\left((p-1) \eta_{t}\right)^{p}}{(T-t)^{p}}+\frac{p}{T-t} \mathcal{Y}_{t}^{\varepsilon, k} \\
& \leq h\left(t, \mathcal{Y}_{t}^{\varepsilon, k}, Z_{t}^{\varepsilon, k}, \phi_{t}^{\varepsilon, k}\right),
\end{aligned}
$$

where we used the Young inequality: $c^{p}+(p-1) y^{q}-p c y \geq 0$ which holds for all $c, y \geq 0$. The comparison theorem implies $Y_{t}^{(k)} \leq \mathcal{Y}_{t}^{\varepsilon, k}$ for all $t \in[0, T-\varepsilon]$ and $\varepsilon>0$.

Recall once again from Condition (B3), then $V_{t, \text {. }}^{\varepsilon, L}$ belongs to $\mathbb{H}^{\varpi}(0, T-\varepsilon)$ for some $\varpi \geq 2$. From the upper bound $Y_{t}^{(k)} \leq k(T+1)$ and from the integrability property of $V_{t, .}^{\varepsilon, k}$, with dominated convergence, by letting $\varepsilon \downarrow 0$ we obtain a.s.

$$
\mathbb{E}\left[\varepsilon^{p} V_{t, T-\varepsilon}^{\varepsilon, k} Y_{T-\varepsilon}^{(k)} \mid \mathcal{F}_{t}\right] \longrightarrow 0
$$

From Assumption (B3), by the proof of Proposition A.1 in [38], there exists a constant $K_{\vartheta, K_{f}, \ell^{\prime}}$ such that a.s.

$$
\mathbb{E}\left[\int_{t}^{T-\varepsilon}\left(V_{t, s}^{\varepsilon, k}\right)^{\frac{\ell^{\prime}}{\ell^{\prime}-1}} d s \mid \mathcal{F}_{t}\right] \leq\left(K_{\vartheta, L_{f}, \ell^{\prime}}\right)^{\left(\ell^{\prime}-1\right) / \ell^{\prime}}
$$


From Conditions (B2) and (B4), it follows that the process $\left((T-t)^{p} b_{t}^{k}, 0 \leq t \leq T\right)$ belongs to $\mathbb{H}^{\ell^{\prime}}(0, T)$ for any $1<\ell^{\prime} \leq \ell$. Therefore by Hölder's inequality we obtain

$$
\mathbb{E}\left[\int_{t}^{T-\varepsilon} V_{t, s}^{\varepsilon, k}(T-s)^{p} b_{s}^{k} d s \mid \mathcal{F}_{t}\right] \leq K_{\vartheta, L_{f}, \ell^{\prime}} \mathbb{E}\left[\int_{t}^{T}\left((T-s)^{p} b_{s}^{k}\right)^{\ell^{\prime}} d s \mid \mathcal{F}_{t}\right]^{1 / \ell^{\prime}} .
$$

Hence we can pass to the limit as $\varepsilon \downarrow 0$

$$
Y_{t}^{(k)} \leq \frac{K_{\vartheta, L_{f}, \ell^{\prime}}}{(T-t)^{p}} \mathbb{E}\left[\int_{t}^{T}\left((T-s)^{p} b_{s}^{k}\right)^{\ell^{\prime}} d s \mid \mathcal{F}_{t}\right]^{1 / \ell^{\prime}}
$$

Assumptions (B2) and (B4) imply by monotone convergence for $k \rightarrow \infty$

$$
Y_{t}^{(k)} \leq \frac{K_{\vartheta, L_{f}, \ell^{\prime}}}{(T-t)^{p}} \mathbb{E}\left[\int_{t}^{T}\left(\left((p-1) \eta_{s}\right)^{p}+(T-s)^{p}\left(f_{s}^{0}\right)\right)^{\ell^{\prime}} d s \mid \mathcal{F}_{t}\right]^{1 / \ell^{\prime}}<+\infty
$$

Using again Hölder's inequality for the conditional expectation, we obtain the upper bound in (1.15).

\section{References}

[1] S. Ankirchner, M. Jeanblanc, and T. Kruse, BSDEs with Singular Terminal Condition and a Control Problem with Constraints, SIAM J. Control Optim. 52 (2014), no. 2, 893-913. MR-3180837

[2] T. M. Apostol, Mathematical analysis, second edition, Addison-Wesley Reading, 1964. MR0344384

[3] P. Bank and M. Voß, Linear quadratic stochastic control problems with stochastic terminal constraint, SIAM J. Control Optim. 56 (2018), no. 2, 672-699. MR-3769694

[4] D. Becherer, Bounded solutions to backward SDE's with jumps for utility optimization and indifference hedging, Ann. Appl. Probab. 16 (2006), no. 4, 2027-2054. MR-2288712

[5] C. Blanchet-Scalliet, A. Cousin, and D. Dorobantu, Hitting time for correlated threedimensional Brownian motion, working paper or preprint, July 2013.

[6] C. Burdzy, Z.-Q. Chen, and J. Sylvester, The heat equation in time dependent domains with insulated boundaries, Journal of mathematical analysis and applications 294 (2004), no. 2, 581-595. MR-2061344

[7] P. Cannarsa, G. Da Prato, and J.-P. Zolesio, The damped wave equation in a moving domain, Journal of Differential Equations 85 (1990), no. 1, 1-16. MR-1052325

[8] R. Cont, Functional Itô calculus and functional Kolmogorov equations, Stochastic integration by parts and functional Itô calculus, Adv. Courses Math. CRM Barcelona, Birkhäuser/Springer, 2016, pp. 115-207. MR-3497715

[9] R. Cont and D.-A. Fournié, A functional extension of the Ito formula, C. R. Math. Acad. Sci. Paris 348 (2010), no. 1-2, 57-61. MR-2586744

[10] F. Cortéz and A. Rodríguez-Bernal, Pdes in moving time dependent domains, Without Bounds: A Scientific Canvas of Nonlinearity and Complex Dynamics, Springer, 2013, pp. 559-577.

[11] F. Delarue, J. Inglis, S. Rubenthaler, and E. Tanré, First hitting times for general nonhomogeneous $1 d$ diffusion processes: density estimates in small time, working paper or preprint, October 2013.

[12] Ł. Delong, Backward stochastic differential equations with jumps and their actuarial and financial applications, European Actuarial Academy (EAA) Series, Springer, London, 2013, BSDEs with jumps. MR-3089193

[13] J. L. Doob, Classical potential theory and its probabilistic counterpart, vol. 262, Springer, 2012. MR-0731258 
[14] B. Dupire, Functional itô calculus, Bloomberg Portfolio Research Paper No. 2009-04FRONTIERS. (2009).

[15] H. Flanders, Differentiation under the integral sign, The American Mathematical Monthly 80 (1973), no. 6, 615-627. MR-0340514

[16] A. Friedman, Partial differential equations of parabolic type, Dover Publications, 2008. MR-0181836

[17] P. Graewe, U. Horst, and E. Séré, Smooth solutions to portfolio liquidation problems under price-sensitive market impact, Stochastic Process. Appl. 128 (2018), no. 3, 979-1006. MR3758345

[18] O. Guéant, The financial mathematics of market liquidity, Chapman \& Hall/CRC Financial Mathematics Series, CRC Press, Boca Raton, FL, 2016, From optimal execution to market making. MR-3586017

[19] S. Iyengar, Hitting lines with two-dimensional brownian motion, SIAM Journal on Applied Mathematics 45 (1985), no. 6, 983-989. MR-0813460

[20] J. Jacod and A. V. Skorohod, Jumping filtrations and martingales with finite variation, Séminaire de Probabilités, XXVIII, Lecture Notes in Math., vol. 1583, Springer, Berlin, 1994, pp. 21-35. MR-1329098

[21] S. G. Kreĭn, Yu. İ. Petunīn, and E. M. Semënov, Interpolation of linear operators, Translations of Mathematical Monographs, vol. 54, American Mathematical Society, Providence, R.I., 1982, Translated from the Russian by J. Szűcs. MR-0649411

[22] T. Kruse and A. Popier, Bsdes with monotone generator driven by brownian and poisson noises in a general filtration, Stochastics 88 (2016), no. 4, 491-539. MR-3473849

[23] T. Kruse and A. Popier, Minimal supersolutions for BSDEs with singular terminal condition and application to optimal position targeting, Stochastic Processes and their Applications 126 (2016), no. 9, 2554 - 2592. MR-3522293

[24] T. Kruse and A. Popier, $L^{p}$-solution for BSDEs with jumps in the case $p<2$ : corrections to the paper 'BSDEs with monotone generator driven by Brownian and Poisson noises in a general filtration., Stochastics 89 (2017), no. 8, 1201-1227. MR-3742328

[25] J. M. Lee, Smooth manifolds, Introduction to Smooth Manifolds, Springer, 2013, pp. 1-31. MR-2954043

[26] M. Marcus and L. Véron, Initial trace of positive solutions of some nonlinear parabolic equations, Comm. Partial Differential Equations 24 (1999), no. 7-8, 1445-1499. MR-1697494

[27] D. Marushkevych and A. Popier, Limit behaviour of the minimal solution of a bsde with singular terminal condition in the non markovian setting, Probability, Uncertainty and Quantitative Risk 5 (2020), no. 1, 1. MR-4068874

[28] A. Metzler, On the first passage problem for correlated brownian motion, Statistics \& probability letters 80 (2010), no. 5-6, 277-284. MR-2593563

[29] B. Øksendal, Stochastic differential equations, Stochastic differential equations, Springer, 2003, pp. 65-84.

[30] E. Pardoux and A. Rascanu, Stochastic Differential Equations, Backward SDEs, Partial Differential Equations, Stochastic Modelling and Applied Probability, vol. 69, Springer-Verlag, 2014. MR-3308895

[31] P. Patie and C. Winter, First exit time probability for multidimensional diffusions: a pde-based approach, Journal of computational and applied mathematics 222 (2008), no. 1, 42-53. MR-2462651

[32] G. Peskir and A. Shiryaev, Optimal stopping and free-boundary problems, Springer, 2006. MR-2256030

[33] A. Popier, Backward stochastic differential equations with singular terminal condition, Stochastic Process. Appl. 116 (2006), no. 12, 2014-2056. MR-2307070

[34] A. Popier, Backward stochastic differential equations with random stopping time and singular final condition, Ann. Probab. 35 (2007), no. 3, 1071-1117. MR-2319716

[35] A. Popier, Limit behaviour of bsde with jumps and with singular terminal condition, ESAIM: PS 20 (2016), 480-509. MR-3581831 
[36] P. E. Protter, Stochastic integration and differential equations, second ed., Applications of Mathematics (New York), vol. 21, Springer-Verlag, Berlin, 2004, Stochastic Modelling and Applied Probability. MR-2020294 MR-2020294

[37] P. E. Protter, Strict local martingales with jumps, Stochastic Processes and their Applications 125 (2015), no. 4, 1352 - 1367. MR-3310350

[38] M.-C. Quenez and A. Sulem, BSDEs with jumps, optimization and applications to dynamic risk measures, Stochastic Process. Appl. 123 (2013), no. 8, 3328-3357. MR-3062447

[39] A. D. Sezer, T. Kruse, and A. Popier, Backward stochastic differential equations with nonMarkovian singular terminal values, Stoch. Dyn. 19 (2019), no. 2, 1950006, 34. MR-3932675 


\section{Electronic Journal of Probability Electronic Communications in Probability}

\section{Advantages of publishing in EJP-ECP}

- Very high standards

- Free for authors, free for readers

- Quick publication (no backlog)

- Secure publication $\left(\mathrm{LOCKSS}^{1}\right)$

- Easy interface (EJMS²)

\section{Economical model of EJP-ECP}

- Non profit, sponsored by $\mathrm{IMS}^{3}, \mathrm{BS}^{4}$, ProjectEuclid ${ }^{5}$

- Purely electronic

\section{Help keep the journal free and vigorous}

- Donate to the IMS open access fund ${ }^{6}$ (click here to donate!)

- Submit your best articles to EJP-ECP

- Choose EJP-ECP over for-profit journals

\footnotetext{
${ }^{1}$ LOCKSS: Lots of Copies Keep Stuff Safe http://www. lockss.org/

${ }^{2}$ EJMS: Electronic Journal Management System http://www.vtex.lt/en/ejms.html

${ }^{3}$ IMS: Institute of Mathematical Statistics http://www.imstat.org/

${ }^{4}$ BS: Bernoulli Society http://www. bernoulli-society.org/

${ }^{5}$ Project Euclid: https://projecteuclid.org/

${ }^{6}$ IMS Open Access Fund: http://www.imstat.org/publications/open.htm
} 